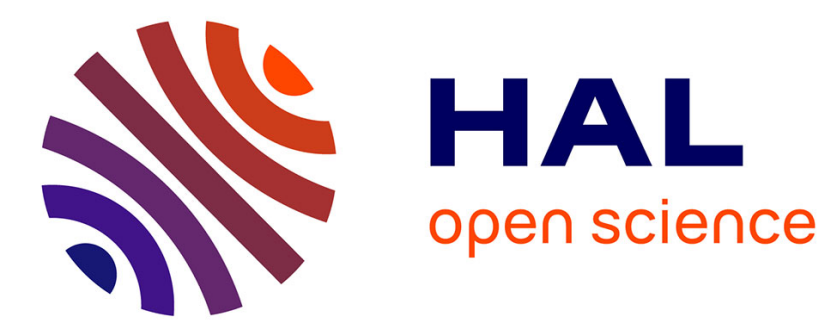

\title{
Acceleration in turbulent channel flow: universalities in statistics, subgrid stochastic models and an application
}

Rémi Zamansky, Ivana Vinkovic, Mikhael Gorokhovski

\section{To cite this version:}

Rémi Zamansky, Ivana Vinkovic, Mikhael Gorokhovski. Acceleration in turbulent channel flow: universalities in statistics, subgrid stochastic models and an application. Journal of Fluid Mechanics, 2013, 721, pp.627-668. 10.1017/jfm.2013.48 . hal-00931506

\section{HAL Id: hal-00931506 https://hal.science/hal-00931506}

Submitted on 17 Jun 2014

HAL is a multi-disciplinary open access archive for the deposit and dissemination of scientific research documents, whether they are published or not. The documents may come from teaching and research institutions in France or abroad, or from public or private research centers.
L'archive ouverte pluridisciplinaire HAL, est destinée au dépôt et à la diffusion de documents scientifiques de niveau recherche, publiés ou non, émanant des établissements d'enseignement et de recherche français ou étrangers, des laboratoires publics ou privés. 


\title{
Acceleration in turbulent channel flow: universalities in statistics, subgrid stochastic models and an application
}

\author{
Rémi Zamanskył, Ivana Vinkovic and Mikhael Gorokhovski $†$ \\ Laboratoire de Mécanique des Fluides et d'Acoustique, Université de Lyon, \\ Université Claude-Bernard Lyon 1/CNRS/Ecole Centrale de Lyon/INSA-Lyon, 69134 Ecully, France
}

(Received 13 March 2012; revised 18 January 2013; accepted 19 January 2013)

This paper focuses on the characterization and the stochastic modelling of the fluid acceleration in turbulent channel flow. In the first part, the acceleration is studied by direct numerical simulation (DNS) at three Reynolds numbers $\left(R e_{*}=u_{*} h / v=180\right.$, 590 and 1000). It is observed that whatever the wall distance is, the norm of acceleration is log-normally distributed and that the variance of the norm is very close to its mean value. It is also observed that from the wall to the centreline of the channel, the orientation of acceleration relaxes statistically towards isotropy. On the basis of dimensional analysis, a universal scaling law for the acceleration norm is proposed. In the second part, in the framework of the norm/orientation decomposition, a stochastic model of the acceleration is introduced. The stochastic model for the norm is based on fragmentation process which evolves across the channel with the wall distance. Simultaneously the orientation is simulated by a random walk on the surface of a unit sphere. The process is generated in such a way that the mean components of the orientation vector are equal to zero, whereas with increasing wall distance, all directions become equally probable. In the third part, the models are assessed in the framework of large-eddy simulation with stochastic subgrid acceleration model (LES-SSAM), introduced recently by Sabel'nikov, Chtab-Desportes \& Gorokhovski (Euro. Phys. J. B, vol. 80, 2011, p. 177-187), and designed to account for the intermittency at subgrid scales. Computations by LES-SSAM and its assessment using DNS data show that the prediction of important statistics to characterize the flow, such as the mean velocity, the energy spectra at small scales, the viscous and turbulent stresses, the distribution of the acceleration can be considerably improved in comparison with standard LES. In the last part of this paper, the advantage of LES-SSAM in accounting for the subgrid flow structure is demonstrated in simulation of particle-laden turbulent channel flows. Compared to standard LES, it is shown that for different Stokes numbers, the particle dynamics and the turbophoresis effect can be predicted significantly better when LES-SSAM is applied.

Key words: intermittency, turbulence modeling, turbulent boundary layers

$†$ Email address for correspondence: mikhael.gorokhovski@ec-lyon.fr

$\ddagger$ Present address: Center for Turbulence Research, Department of Mechanical Engineering, Stanford University, Stanford, CA 94305, USA. 


\section{Introduction}

Beyond the traditional statement that channel flow is driven by the pressure gradient working against the wall friction, recent studies at high Reynolds number revealed an intriguing complexity of such a flow (see recent reviews by Marusic et al. 2010, Smits, McKeon \& Marusic 2011 or Jiménez 2012). The origin of this complexity stems from the interactions between multiscale intermittent coherent motions. A significant role is attributed to the viscous layer, in which most of the turbulent kinetic energy is produced in tiny structures, comparable with the dissipative length scales. When the Reynolds number is high, the direct numerical resolution of these scales is hampered by limitations of computational resources. In the meantime, applying the standard large-eddy simulation (LES) approach at high Reynolds number, the momentum transfer across the channel remains 'under-resolved', and the wall shear stress is inaccurately predicted (Piomelli \& Balaras 2002). In order to account for the effects of the discarded scales, various ideas and analytical frameworks have been proposed. Examples include two widely used approaches: hybrid Reynolds-averaged Navier-Stokes (RANS)/LES methods (Piomelli et al. 2003; Spalart et al. 2006; Larsson, Lien \& Yee 2007; Shur et al. 2008; Chaouat \& Schiestel 2009) and LES combined with a subgrid-scale (SGS) model (Kerstein 1999, 2002; Piomelli \& Balaras 2002; Sagaut 2002; Schmidt, Kerstein \& Wunsch 2003; Westbury, Dunn \& Morrison 2004; Burton \& Dahm 2005a,b; Kemenov \& Menon 2006, 2007; Park \& Mahesh 2008).

In this paper, we consider the second approach. Westbury et al. (2004) propose simulating stochastically the residual stress tensor in the boundary layer. Their model focuses on the reverse transfers of energy related to interactions between structures of different sizes. Schmidt et al. (2003) propose stochastically estimating the nearwall velocity fluctuations with a one-dimensional equation of turbulence (Kerstein $1999,2002)$. In the approach of Kemenov \& Menon $(2006,2007)$ the instantaneous velocity field is decomposed into large-scale and small-scale parts. The small-scale motions are simulated by the simplified one-dimesional momentum equation (in three directions), and the modelled subgrid stress is coupled with large-scale equations. These and other SGS models are well described and examined, and we do not dwell on them further. Most important is that most of the SGS models (Meneveau \& Katz 2000) are focused on statistics of the residual velocity field. Customarily in such models, the amplitude of the velocity fluctuations is not directly linked to the Reynolds number, which is known to be an essential parameter of the small-scale flow structure. This motivated Burton \& Dahm $(2005 a, b)$ to combine LES with a multifractal model of the small-scale vorticity field, which scales with the local Reynolds number. They show that for homogeneous isotropic turbulence (HIT), the model provides an intermittent velocity field in good agreement with statistics from direct numerical simulations (DNS).

A natural alternative is to introduce the total acceleration $\left(a_{i}=\partial_{t} u_{i}+u_{j} \partial_{j} u_{i}\right)$ as a key variable of the SGS model. This variable directly represents the resultant of forces applied on the fluid and is essentially determined by the small-scale structures of the flow. An SGS model based on the acceleration was proposed recently by Sabel'nikov, Chtab-Desportes \& Gorokhovski (2011), with applications to LES of HIT. Along with improving the small-scale part of the energy spectra, this approach allows prediction of the effects of intermittency reported earlier in the experimental studies of Mordant et al. (2002) and Mordant, Crawford \& Bodenschatz (2004a)

In this paper, our motivation is to study the statistics of the acceleration for channel flow and to construct an SGS model based on this variable. Only a few investigations 
highlight the behaviour of the acceleration in channel flow (Christensen \& Adrian 2002; Choi, Yeo \& Lee 2004; Lee, Yeo \& Choi 2004; Chen et al. 2010; Yeo, Kim $\&$ Lee 2010). It is shown that acceleration is a strongly intermittent variable. As a signature of its intermittency, the probability density function (p.d.f.) of acceleration shows stretched tails (Choi et al. 2004). This intermittency is generally linked to the dynamics of vortical near-wall structures. The strongest intermittency is observed in the viscous wall layer, and is attributed to effects of near-wall streaks (Lee et al. 2004; Yeo et al. 2010). Once the fluid-particle path is linked to a tiny helical motion, its centripetal acceleration is characterized by large fluctuations. Around such a structure, the acceleration direction changes rapidly, meanwhile its norm is preserved during the structure lifetime. This leads to the long-time correlation of the acceleration norm, versus the short time correlation of its orientation (of the order of the Kolmogorov time). Previously, this was observed in high-Reynolds-number HIT: see experiments by Voth, Satyanarayan \& Bodenschatz (1998), Voth et al. (2002), Sawford et al. (2003), Mordant, Crawford \& Bodenschatz (2004b) and Xu et al. (2007), numerical simulations by Pope (1990), Vedula \& Yeung (1999), Tsinober, Vedula \& Yeung (2001), Sawford et al. (2003) and Yeung et al. (2006) as well as development of models by Monin \& Yaglom (1981), Pinsky, Khain \& Tsinober (2000), Hill (2002), Sawford et al. (2003) and Lamorgese et al. (2007); see also the review of Toschi \& Bodenschatz (2009). Consequently, for HIT there is an underlying suggestion of time-scale separation of the norm and orientation of acceleration (Pope 1990; Mordant et al. 2002, 2004a; Sabel'nikov et al. 2011):

$$
a_{i}=|a| e_{i},
$$

where $|a|$ is the acceleration norm and $e_{i}$ is its orientation. In Sabel'nikov et al. (2011), the two variables, $|a|$ and $e_{i}$ are stochastically simulated as independent processes. They showed that assumption (1.1) along with temporal correlation of orientation $e_{i}$ on the Kolmogorov time scale is consistent with the Kolmogorov-Oboukhov 41 theory.

In our paper, along with the assumption of the decomposition (1.1), the modelling of the acceleration for turbulent channel flow takes into account the two following observations (Chen et al. 2010; Yeo et al. 2010): (i) the contribution of the longitudinal solenoidal component is non-negligible in the viscous wall region; (ii) tiny streaky motions close to the wall interact with the coherent structures of the outer zone. However, to our knowledge the statistical universalities that may be attributed to the acceleration norm and orientation across turbulent channel flow remain unknown. This motivated us to perform DNS of turbulent channel flow, and to analyse the scaling of $|a|$ and $e_{i}$ with the wall distance and the Reynolds number. In the framework of Barenblatt's complete and incomplete similarities, we propose a universal profile for the mean norm of acceleration across the channel. Further, with our DNS, we observe that the acceleration norm presents a log-normal distribution whatever the wall distances are, while its orientation manifests return to isotropy away from the wall. In line with these observations, we propose to model the acceleration norm by fragmentation processes under scaling symmetry evolving with the distance from the wall. For the acceleration orientation, the evolution toward statistical isotropy is simulated by a random walk on the unit sphere.

The objective is to assess these models for the computation of high-Reynoldsnumber channel flow. To this end, our new stochastic models are coupled with the Navier-Stokes equations in the large-eddy simulation with stochastic subgrid acceleration model (LES-SSAM) framework of Sabel'nikov et al. (2011). Finally, LES-SSAM is also assessed in turbulent channel flow with immersed solid inertial 


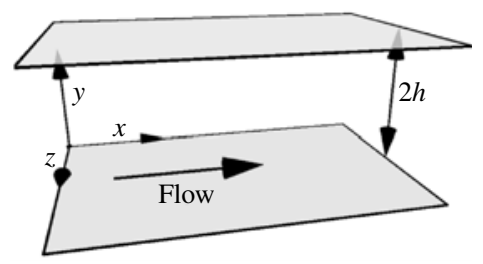

FIGURE 1. Channel flow. $x, y, z$ represent the streamwise, the wall-normal and the spanwise directions respectively.

point particles. In such a practically important flow, the particles interact with turbulence at small scales, and hence, access to the flow structure at unresolved scales is of primary importance for predictive simulations of the particle dynamics.

This paper is organized as follows. After a brief description of the numerical approach in $\S 2$, we focus in $\S 3$ on universality in scaling of the acceleration norm and orientation. In $\S 4$, the new stochastic models are proposed and assessed by DNS for the acceleration norm and orientation. The application is given in $\S 5$, where in the framework of LES-SSAM, the new stochastic models are assessed by comparison with DNS and standard LES. At the end of $\S 5$, this assessment is extended to channel flows with inertial particles. The particle dynamics and the turbophoresis effect are analysed in the framework of LES-SSAM, DNS and standard LES.

\section{Details of the numerical simulations}

Fully developed incompressible turbulent channel flow driven by a constant mean pressure gradient is considered here. The flow is statistically homogeneous in the streamwise $(x)$ and the spanwise $(z)$ directions and it is inhomogeneous in the wallnormal direction $(y)$ as illustrated in figure 1 . The channel height is $2 h$. This flow is given by the continuity and the Navier-Stokes equations:

$$
\begin{aligned}
\nabla \cdot \boldsymbol{u} & =0, \\
\frac{\partial \boldsymbol{u}}{\partial t}+(\boldsymbol{u} \cdot \nabla) \boldsymbol{u} & =-\frac{1}{\rho} \nabla P+v \nabla^{2} \boldsymbol{u},
\end{aligned}
$$

where $v$ is the kinematic viscosity and $\rho$ the fluid density. Periodic boundary conditions are imposed in the $x$ - and $z$-directions. At the wall, the no-slip boundary condition is applied:

$$
\boldsymbol{u}(y=0)=\boldsymbol{u}(y=2 h)=0 .
$$

Equations (2.1)-(2.3) are solved using a Galerkin spectral approximation (Fourier Chebyshev) and the variational projection method in a divergence-free space. This method is based on the original approach of Moser, Moin \& Leonard (1983). The further development of this method given by Buffat, Le Penven \& Cadiou (2011) was used with rotational form for convective terms in order to ensure the energy conservation. For the computation of the Fourier transform of the nonlinear term the $3 / 2$ de-aliasing rule is applied. The time integration uses the explicit Adams-Bashforth algorithm for the nonlinear terms, and the semi-implicit algorithm for the diffusion terms. This method was further tested in Zamansky, Vinkovic \& Gorokhovski (2010), by comparison of the velocity statistics in channel flow with the DNS of Moser, Kim \& Mansour (1999) and Hoyas \& Jiménez (2008). 

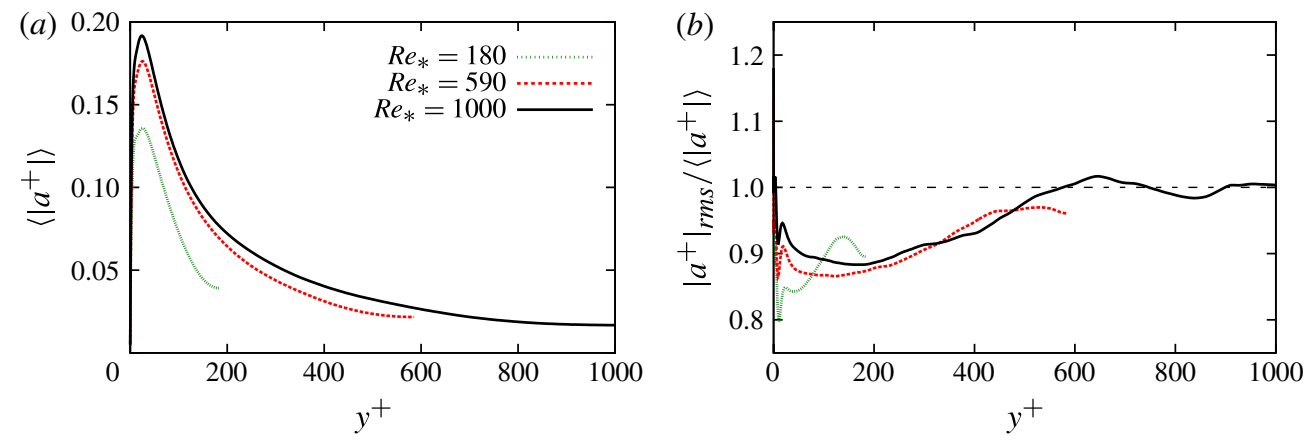

Figure 2. (Colour online) Profiles of the mean $(a)$ and of the variance-to-mean ratio $(b)$ of the acceleration norm for $R e_{*}=180,590$ and 1000. Acceleration is normalized by $a_{*}=u_{*}^{3} / \nu$.

$$
\begin{array}{cccccc}
R e_{*} & R e_{c} & N_{x} \times N_{y} \times N_{z} & L_{x} \times L_{y} \times L_{z} & \Delta x^{+} \times \Delta y^{+} \times \Delta z^{+} & \mathrm{d} t^{+} \\
180 & 3280 & 192 \times 193 \times 192 & 3 \pi h \times 2 h \times \frac{4}{3} \pi h & 9.0 \times(0.02 \sim 3.0) \times 4.0 & 0.030 \\
587 & 12490 & 384 \times 257 \times 384 & \frac{3}{2} \pi h \times 2 h \times \frac{3}{4} \pi h & 7.2 \times(0.04 \sim 7.2) \times 3.6 & 0.033 \\
1000 & 22250 & 512 \times 385 \times 512 & \frac{4}{3} \pi h \times 2 h \times \frac{2}{3} \pi h & 8.2 \times(0.03 \sim 8.3) \times 4.1 & 0.034
\end{array}
$$

TABLE 1. Parameters of the DNS. $N_{i}$ and $L_{i}$ are the number of grid points and the domain length in direction $i . \Delta x^{+}, \Delta y^{+}$and $\Delta z^{+}$are the typical grid spacings and $\mathrm{d} t^{+}=\mathrm{d} t / t_{*}$ is the time step.

Parameters of the present DNS are given in table 1 , where $R e_{*}=u_{*} h / v$ is the Reynolds number based on the friction velocity $u_{*}$, while $R e_{c}=u_{c} h / v$ is the Reynolds number based on the mean channel-centre velocity $\left(u_{c}\right)$. In the following, the superscript ' + ' will denote quantities normalized by $u_{*}, v$ and $\rho$.

\section{Statistics of acceleration}

This section focuses on the evolution of the acceleration statistics with the wall distance and the Reynolds number. In the first part we consider the statistics of the acceleration norm. In the second part, the orientation of the acceleration vector is studied, and finally we discuss the statistical independence of those two quantities. The statistical properties observed by DNS are further used in $\S 4$ for construction of an SGS model for acceleration.

\subsection{Norm of acceleration: log-normality and scaling properties}

For three Reynolds numbers: $R e_{*}=180,590$ and 1000, the profiles from our DNS of the mean and the standard deviation of acceleration norm $|a|=\left(a_{i} a_{i}\right)^{1 / 2}$ are given in figure 2. It is seen that the acceleration norm is characterized by a peak close to the wall, and that the profiles depend on the Reynolds number. In figure $2(b)$, the ratio between the root-mean-square (r.m.s.) and the mean of the acceleration norm is shown. This ratio stays relatively close to unity and tends to stay closer to unity with increasing the distance to the wall and the Reynolds number. Hence for the 
acceleration norm we conclude that approximately:

$$
|a|_{r m s} \equiv\left(\left\langle|a|^{2}\right\rangle-\langle|a|\rangle^{2}\right)^{1 / 2} \approx\langle|a|\rangle .
$$

This is consistent with the dominant role of the fluctuations in the statistics of acceleration components observed by Chen et al. (2010), even very close to the wall.

Let us examine now the p.d.f. shape of $\xi=|a| /|a|_{r m s}$. By definition, the log-normal distribution of $\xi$ is given by:

$$
\mathscr{L} \mathscr{N}\left(\xi ; \mu, \sigma^{2}\right)=\frac{1}{\xi \sqrt{2 \pi \sigma^{2}}} \exp \left(-\frac{(\ln \xi-\mu)^{2}}{2 \sigma^{2}}\right)
$$

with parameters $\mu$ and $\sigma^{2}$. When approximation (3.1) is used, along with the expression of moments of the log-normal distribution $\left(m_{k}=\exp \left(k \mu+k^{2} \sigma^{2} / 2\right)\right)$, we have:

$$
\begin{aligned}
\sigma^{2} & =\ln 2, \\
\mu & =-\frac{1}{2} \ln 2 .
\end{aligned}
$$

It is interesting to compare (3.2)-(3.4) with the one-point p.d.f. of $\xi=|a| /|a|_{\text {rms }}$ obtained by the DNS data at different wall-normal distances, and at different Reynolds numbers. This is shown in figure 3. It is seen that the p.d.f. shape remains almost invariant with respect to the wall distance for all three Reynolds numbers. It is also seen that the log-normal distribution (3.2)-(3.4) is very close to the DNS distributions, even in the near-wall region. Besides, there is no appreciable departure from the lognormality in the small sample space of strong statistical fluctuations. The log-normality of the acceleration statistics has already been observed in HIT, by Yeung et al. (2006) from DNS and experimental studies of Mordant et al. (2004b) and Reynolds et al. (2005). Also it is stressed in these papers that the behaviour of the statistics of the acceleration modulus may be different from those of the acceleration components. Measurements of Mordant et al. (2004b) have shown, for HIT, that the shape of the p.d.f. for the norm exhibits invariance with the Reynolds number whereas the p.d.f.s of the components are Reynolds-number dependent. The log-normality of the acceleration norm, observed here by DNS, will be assumed in $\S 4.1$ of this paper for the construction of a stochastic model for this variable.

Also interesting is the following question: how may the mean norm of acceleration be scaled at a given distance from the wall? As a local reference acceleration scale, we use the local Kolmogorov acceleration $a_{\eta}=\langle\varepsilon\rangle^{3 / 4} v^{-1 / 4}(\langle\varepsilon\rangle$ is the local mean dissipation rate). Under the classical assumption, the flow properties at a given point are specified by the wall shear stress $\tau_{w}$, the kinematic viscosity $v$, the fluid density $\rho$, the distance to the wall $y$ and the channel height $h$. Using these physical quantities, dimensional analysis gives:

$$
\begin{aligned}
\frac{\langle|a|\rangle}{a_{*}} & =\Pi_{a}\left(y^{+}, R e_{*}\right), \\
\frac{\langle\varepsilon\rangle}{\varepsilon_{*}} & =\Pi_{\varepsilon}\left(y^{+}, R e_{*}\right),
\end{aligned}
$$

where $a_{*}=u_{*}^{3} / \nu$ and $\varepsilon_{*}=u_{*}^{4} / \nu$ are characteristic acceleration and dissipation wall scales. $\Pi_{a}$ and $\Pi_{\varepsilon}$ are two universal dimensionless functions with global and local 

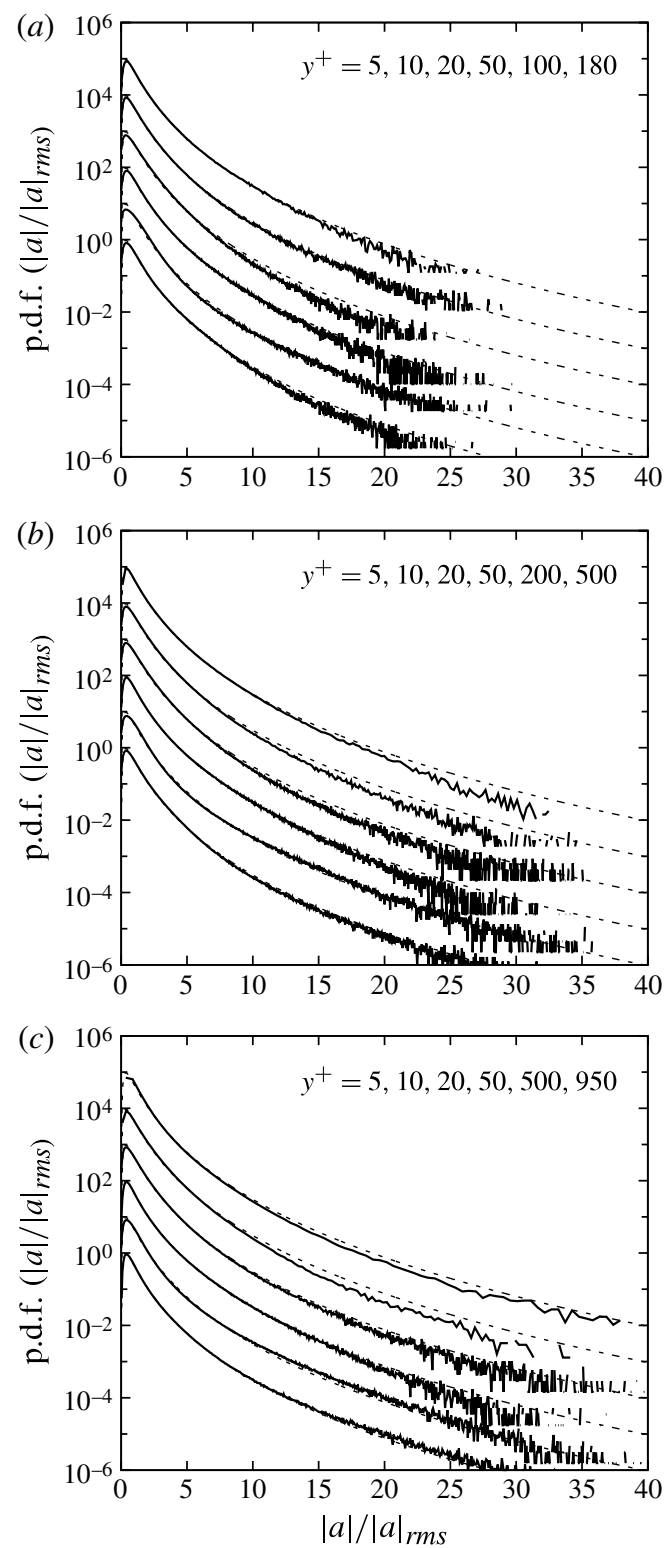

FIGURE 3. p.d.f.s for the norm of acceleration for $R e_{*}=180(a), 590(b)$, and $1000(c)$ and for several distances to the wall, each shifted upward by one decade, with the bottom line corresponding to the lowest value of $y^{+}$. The DNS data are compared with the log-normal distribution with $\mu=-\sigma^{2} / 2$ and $\sigma^{2}=\ln (2)$ (dashed lines).

parameters $R e_{*}$ and $y^{+}$, respectively. Using the Kolmogorov scaling for the local acceleration, the combination of (3.5) and (3.6) leads to:

$$
\frac{\langle|a|\rangle}{a_{\eta}}=\Pi_{a}^{\prime}\left(y^{+}, R e_{*}\right),
$$



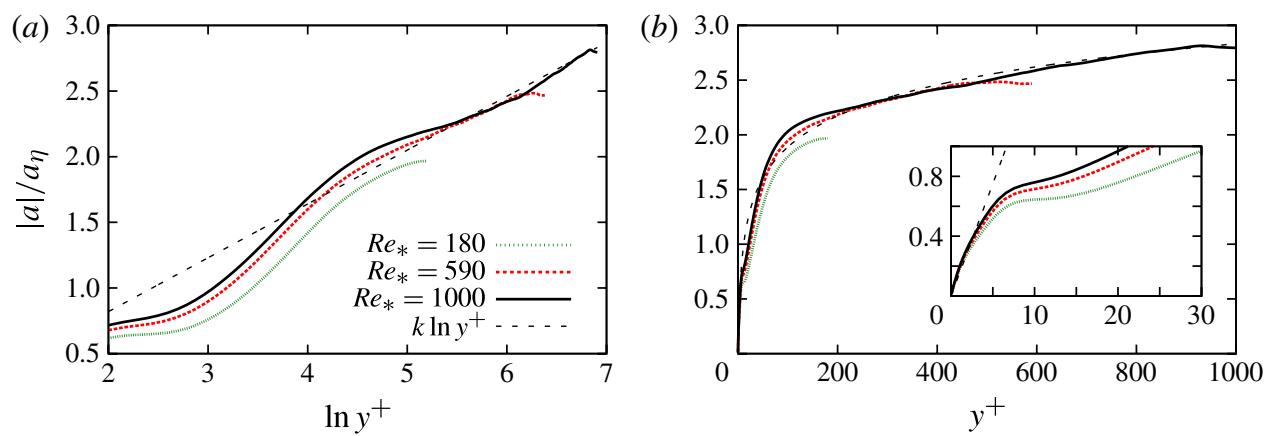

FIguRE 4. (Colour online) Comparison for $R e=180,590$ and 1000 between $\langle|a|\rangle / a_{\eta}$ and the log-law (3.11). (a) Semi-logarithmic scale and (b) linear scale; inset: zoom in the nearwall region and comparison with the linear curve $0.15 y^{+}$.

where $\Pi_{a}^{\prime}=\Pi_{a} / \Pi_{\varepsilon}^{3 / 4}$ is also a universal dimensionless function. Differentiating (3.7) with respect to $y^{+}$, it may be written:

$$
y^{+} \frac{\partial}{\partial y^{+}} \frac{\langle|a|\rangle}{a_{\eta}}=y^{+} \frac{\partial}{\partial y^{+}} \Pi_{a}^{\prime}\left(y^{+}, R e_{*}\right)=\Pi_{a}^{\prime \prime}\left(y^{+}, R e_{*}\right) .
$$

The DNS data from Lee et al. (2004), along with our DNS, show that the contribution of viscous forces to the acceleration is dominant only in the viscous region $\left(y^{+}<30\right)$. For larger distances from the wall, the solenoidal component of acceleration is much smaller than its potential component. One may assume that for high enough $R e$ and $y$ there is a region far from the wall and far from the centreline where viscosity no longer influences the acceleration. Then, in the framework of complete similarity (Barenblatt 1996) with respect to $R e_{*}$ and $y^{+}$, and following the von Kármán hypothesis of the vanishingly small effect of viscosity, it may be supposed that $\Pi_{a}^{\prime \prime}\left(y^{+} \rightarrow \infty, R e \rightarrow \infty\right)$ adopts a constant value $k$ :

$$
y^{+} \frac{\partial}{\partial y^{+}} \frac{\langle|a|\rangle}{a_{\eta}}=\Pi_{a}^{\prime \prime}(\infty, \infty)=k .
$$

This leads to the logarithmic profile:

$$
\frac{\langle|a|\rangle}{a_{\eta}}=k \ln y^{+}+A .
$$

We estimate constants $k$ and $A$ by DNS. The best fit of (3.10) to DNS data gives: $A=0$ and $k \approx 0.41$ (surprisingly very close to the von Kármán constant $\kappa$, although for the mean velocity profile, one finds $1 / \kappa$ ), i.e.

$$
\frac{\langle|a|\rangle}{a_{\eta}}=\kappa \ln y^{+} .
$$

In figure 4 , the evolution of $\langle|a|\rangle / a_{\eta}$ from DNS is compared with (3.11) for three Reynolds numbers. It is seen that in the outer layer $\left(y^{+}>50\right)$ the local mean norm of acceleration scaled by the local mean Kolmogorov acceleration follows approximately the logarithmic profile (3.11). Very close to the wall $\left(y^{+}<5\right)$, the quantity $\langle|a|\rangle / a_{\eta}$ from DNS is linear (see inset in figure $4 b$ ). The transition region between the linear and the logarithmic behaviour takes place for $5<y^{+}<50$. We note here that in 

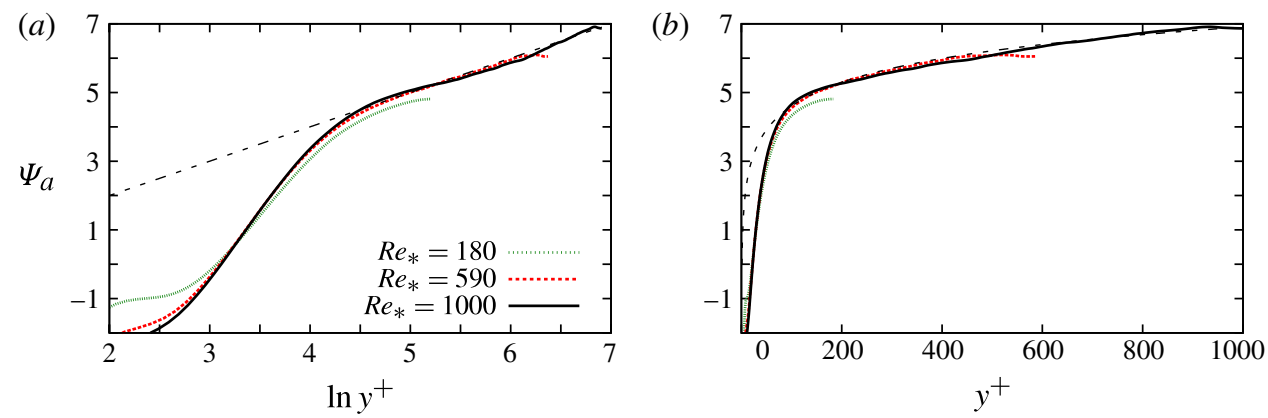

FIGURE 5. (Colour online) Comparison of $\langle|a|\rangle / a_{\eta}$, in the plane $\left(\Psi_{a}=\right.$ $\left.\left(\ln R e / \beta_{1}\right) \ln \left(\langle|a|\rangle / a_{\eta} /\left(D_{0} \ln R e+D_{1}\right)\right) ; \ln y^{+}\right)$between DNS for $\operatorname{Re}_{*}=180,590$ and 1000 , and (3.13) with $\beta_{1}=1, D_{0}=0.15$ and $D_{1}=0.01$ (dashed line). (a) Log-linear scale $(b)$ linear scale.

Yeo et al. (2010), the linear behaviour of $\langle|a|\rangle / a_{\eta}$ in the inertial range with $y^{+}$was proposed, with a slope depending on the Reynolds number.

An alternative physics in the estimation of (3.8) can be provided by assuming that the viscosity has a small but not vanishing effect across the entire channel. In line with Barenblatt's incomplete similarity with respect to $y^{+}$and the lack of self-similarity with respect to $R e_{*}$ (Barenblatt 1993), the following general form may be suggested in the absence of a characteristic length of the flow:

$$
\frac{\partial}{\partial y^{+}} \frac{\langle|a|\rangle}{a_{\eta}}=\frac{1}{y^{+}} B(R e)\left(y^{+}\right)^{\beta(R e)} .
$$

If $R e \rightarrow \infty$, the expansion of functions $\beta(R e)$ and $B(R e)$ in powers of a small parameter $1 / \ln \operatorname{Re}$ leads to

$$
\frac{\langle|a|\rangle}{a_{\eta}}=\left(D_{0} \ln R e+D_{1}\right)\left(y^{+}\right)^{\beta_{1} / \ln (R e)} .
$$

Coefficients $\beta_{1}, D_{0}$ and $D_{1}$ can be estimated from DNS as proposed by Barenblatt $\&$ Prostokishin (1993). For the Reynolds number based on the centreline velocity $R e=R e_{c}$, it is found that $\beta_{1}=3 / 2, D_{0}=0.10$ and $D_{1}=0.02$; for the Reynolds number based on the friction velocity $R e=R e_{*}$, it is found that $\beta_{1}=1, D_{0}=0.15$ and $D_{1}=0.01$. The values found here for exponent $\beta_{1}$ by DNS are identical to those proposed by Barenblatt (1993) for the mean velocity profile. In figure 5, (3.13) with suggested constants $\beta_{1}, D_{0}$ and $D_{1}$ is plotted against DNS data in the plane of the following coordinates: $\left(\Psi_{a}=\left(\ln R e / \beta_{1}\right) \ln \left(\langle|a|\rangle / a_{\eta} /\left(D_{0} \ln R e+D_{1}\right)\right) ; \ln y^{+}\right)$. It is seen that for $y^{+}>20$, the DNS of $\langle|a|\rangle / a_{\eta}$ for all three Reynolds numbers collapse onto a single curve. Also, for $y^{+}>100$, the profiles of $\langle|a|\rangle / a_{\eta}$ for the three Reynolds numbers are quite well fitted by the power-law function (3.13). The latter is presented by the first bisectrix $\left(\Psi_{a}=\ln y^{+}\right)$in figure 5(a). Compared to figure 4, in which the DNS data were fitted by the logarithmic profile (3.11), it is seen that the power law (3.13) represents a slightly better fit of the DNS data. This is consistent with the non-vanishing effect of viscosity across the whole channel, at least for this range of Reynolds number. 

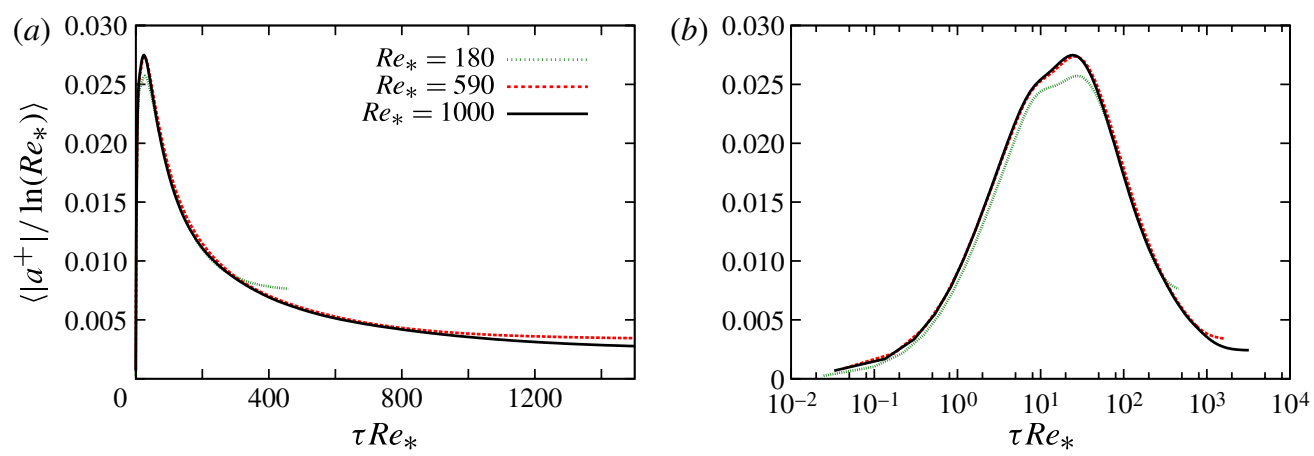

FIGURE 6. (Colour online) Almost universal profile of normalized mean norm of acceleration as a function of parameter $\tau^{+}=R e_{*} \ln (h /(h-y))$ for three Reynolds numbers: (a) linear scale and $(b)$ semi-logarithmic scale.

In (3.13), the ratio $D_{1} / D_{0}$ is small compared to $\ln R e_{*}$. Then this equation may be simplified as:

$$
\frac{\langle|a|\rangle}{a_{*} \ln R e_{*}}=\frac{a_{\eta}}{a_{*}} D_{0}\left(y^{+}\right)^{\beta_{1} / \ln R e_{*}} .
$$

Recalling here the Kolmogorov scaling of acceleration $a_{\eta}$ and definitions of $a_{*}$ and $\varepsilon_{*}$, using an estimation from Landau \& Lifshitz (1987) $\langle\varepsilon\rangle=\varepsilon_{*} \partial\left\langle u^{+}\right\rangle / \partial y^{+}$, and assuming again Barenblatt-type incomplete similarity (Barenblatt 1996): $\partial\left\langle u^{+}\right\rangle / \partial y^{+}=$ $\left(y^{+}\right)^{a} \Phi\left(R e_{*}\right)$, one may rewrite (3.14) in the following form:

$$
\frac{\langle|a|\rangle}{a_{*} \ln R e_{*}}=D_{0}\left(y^{+}\right)^{3 \zeta / 4+\beta_{1} / \ln R e_{*}} \Phi\left(R e_{*}\right),
$$

where the exponent $\zeta$ is also assumed to be a function of $R e_{*}$. In spite of this complex dependence on $R e_{*}$ provided by the similarity analysis, one may ask: is it possible to associate a self-similarity argument to $\langle|a|\rangle / a_{*} \ln R e_{*}$, such that this quantity becomes universal across the whole domain of $y^{+}$for all three Reynolds numbers $R e_{*}=180$, 590 and 1000? To this end, we introduce the following evolution parameter:

$$
\tau=\ln \left(\frac{h}{h-y}\right)=-\ln \left(1-\frac{y^{+}}{R e_{*}}\right) .
$$

This parameter is equal to zero at the wall: $y=0, \tau=0$, and tends to infinity when the wall-normal distance reaches the external flow scale - the channel centreline here: $y \rightarrow h, \tau \rightarrow \infty$. The logarithmic form of this parameter is convenient for the representation of long-distance influences of the wall. Further motivations and references for the choice of this parameter are provided in $\S 4$.

In figure 6 , the DNS data are plotted against $\tau^{+}=\tau R e_{*}$. It is seen that for the three Reynolds numbers, profiles $\langle|a|\rangle / a_{*} \ln R e_{*}$ from DNS collapse almost on a single curve up to approximately $y / h=1-\exp \left(-\tau^{+} / R e_{*}\right)<0.85$. This supports to some extent the universality of the introduced coordinates:

$$
\left(\frac{a}{a_{*} \ln R e_{*}} ; \tau^{+}\right) .
$$




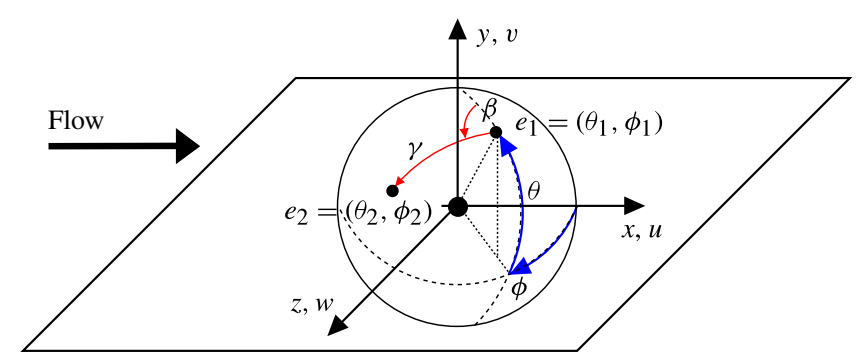

FIGURE 7. (Colour online) Coordinate system, and definition of angles $\phi, \theta, \gamma$ and $\beta$.

\subsection{Orientation of acceleration: return to isotropy}

As shown schematically in figure 7 , the acceleration orientation $e_{i}=a_{i} /|a|$ is defined on the unit sphere by two angles denoted here as 'longitude' $\phi$ and 'latitude' $\theta$. These angles lie in two orthogonal planes: $\phi(-\pi<\phi \leqslant \pi)$ in the plane $(x, z)$ parallel to the wall, and $\theta(-\pi / 2 \leqslant \theta \leqslant \pi / 2)$ in the normal-to-wall plane $(x, y) . \theta=0$ and $\theta= \pm \pi / 2$ correspond to alignment with the wall and to the wall-normal directions, respectively. Each component of the orientation is then given by:

$$
e_{i}=\frac{a_{i}}{|a|}=\left\{\begin{array}{l}
e_{x}=\cos (\theta) \cos (\phi) \\
e_{y}=\sin (\theta) \\
e_{z}=\cos (\theta) \sin (\phi) .
\end{array}\right.
$$

With these definitions, the p.d.f.s of $\phi$ and $\theta$ corresponding to a statistically isotropic orientation are:

$$
\begin{aligned}
& P_{\phi, \text { iso }}(\phi)=\frac{1}{2 \pi}, \quad-\pi<\phi \leqslant \pi, \\
& P_{\theta, \text { iso }}(\theta)=\frac{|\cos (\theta)|}{2}, \quad-\frac{\pi}{2} \leqslant \theta \leqslant \frac{\pi}{2} .
\end{aligned}
$$

Corresponding to these distributions the mean of $\sin \phi$ and $\sin \theta$ is zero and the variance is given by:

$$
\begin{aligned}
& \left\langle\sin (\phi)^{2}\right\rangle_{i s o}=\int_{-\pi}^{\pi} \sin (\phi)^{2} P_{\phi, i s o}(\phi) \mathrm{d} \phi=\frac{1}{2}, \\
& \left\langle\sin (\theta)^{2}\right\rangle_{i s o}=\int_{-\pi / 2}^{\pi / 2} \sin (\theta)^{2} P_{\theta, i s o}(\theta) \mathrm{d} \theta=\frac{1}{3} .
\end{aligned}
$$

In figure 8 , the variances of $\sin \phi$ and $\sin \theta$ are compared to (3.21) and (3.22) for $R e_{*}=180,590$ and 1000. In figure $8(a)$, it can be seen that across the channel, the deviation of $\left\langle\sin (\phi)^{2}\right\rangle$ from $\left\langle\sin (\phi)^{2}\right\rangle_{\text {iso }}$ given by (3.21) is quite small. Concerning the DNS of $\left\langle\sin (\theta)^{2}\right\rangle$ (see figure $8 b$ ), in the inner layer, the variance increases almost linearly with the wall distance until $y^{+} \approx 20$. For intermediate distance to the wall (say $y^{+}>50$ and $\left.y / h<0.3\right),\left\langle\sin (\theta)^{2}\right\rangle$ is shown to be very close to the isotropic orientation value (3.22). This intermediate range seems to correspond to the actual domain of validity of the logarithmic law of the velocity profile (Pope 2000). For larger distances to the wall, the variance of $\sin \theta$ presents an $R e_{*}$-dependence but stays approximately close to its value for isotropic orientation. Finally, note that the orientation is not well defined when the acceleration is zero. This prevents observation of the orientation too 

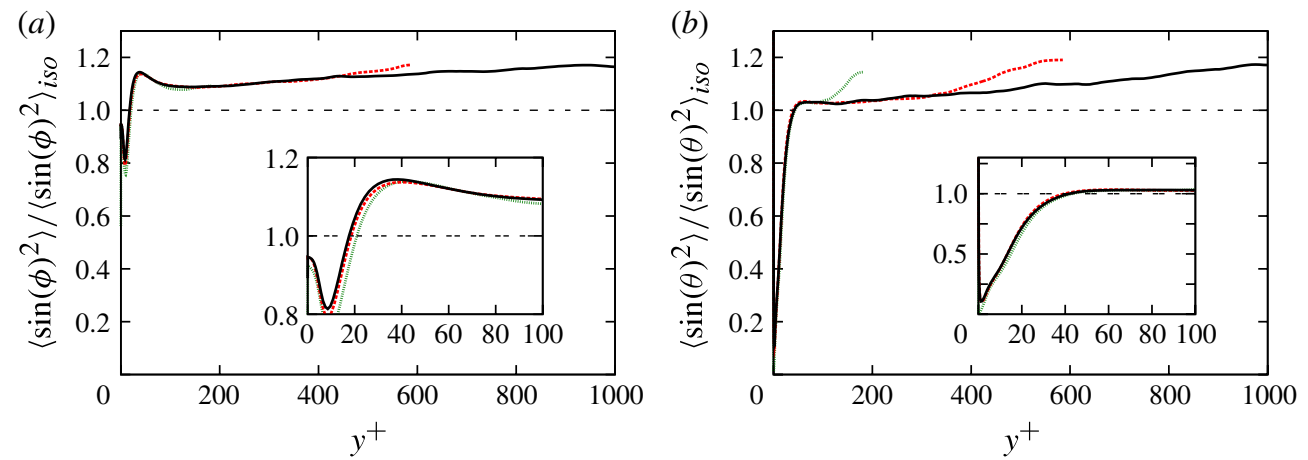

Figure 8. (Colour online) Profile of the variance of $\phi(a)$ and $\theta(b)$ for $\operatorname{Re}_{*}=180,590$ and 1000 (same line styles as previous figures). Variance of $\phi$ and $\theta$ is normalized by their values in the case of isotropy: (3.21) and (3.22). Inset: zoom in the near-wall region $\left(y^{+}<100\right)$.
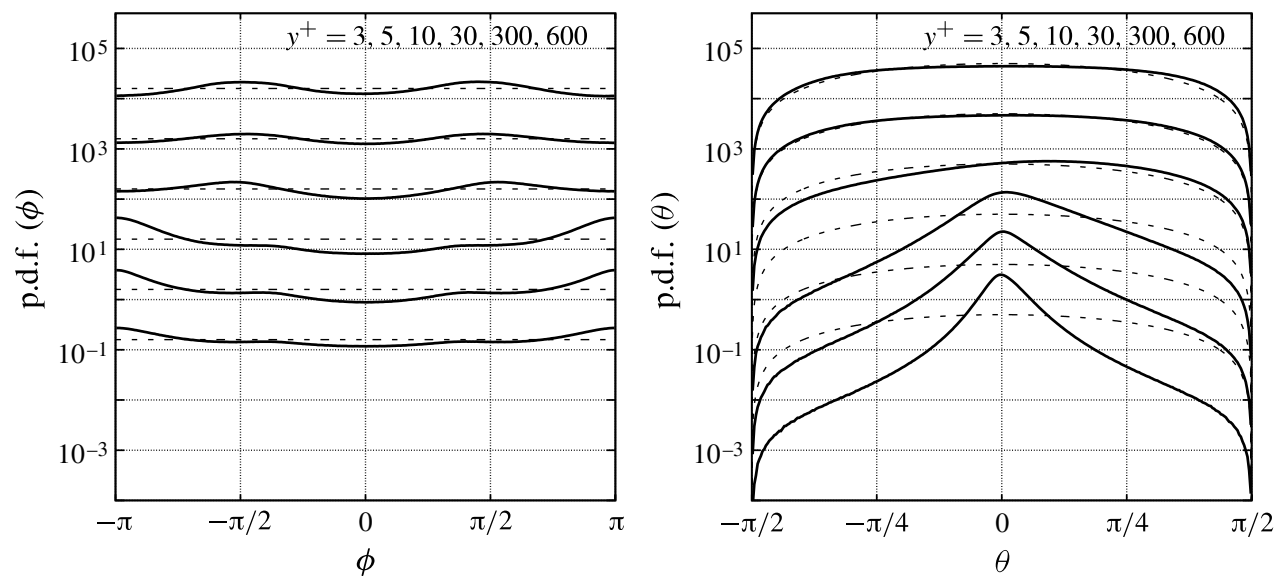

FIGURE 9. p.d.f.s of $\phi(a)$ and of $\theta(b)$ at different distances to the wall (each shifted by one decade upward, with the bottom line corresponding to the lowest value of $y^{+}$). DNS for $R e_{*}=1000$ (continuous lines), and comparison with the isotropic p.d.f.s (dashed lines) $P_{\phi, \text { iso }}$ and $P_{\theta, i s o},(3.19)$ and (3.20).

close to the wall (say $y^{+}<1$ ). To some extent, this relaxation towards isotropy is confirmed by the DNS of Antonia, Kim \& Browne (1991). In their study, the classical hypothesis of local isotropy of the small-scale motions in turbulent channel flow $\left(R e_{*}=180\right.$ and 395) was assessed by invariants of the velocity-derivative tensor. It is found that very close to the wall, the turbulent structures are almost bi-dimensional. The isotropy in the small-scale motions is observed by those authors only near the centre of the channel.

In figure 9, the p.d.f.s of both $\phi$ and $\theta$ are shown for $R e_{*}=1000$ at different distances to the wall. It is seen that $P(\phi)$ (figure $9 a$ ) does not vary appreciably from $P_{\phi, i s o}(\phi)$ given by (3.19). Nevertheless, it may be observed that very close to the wall $\left(y^{+}<10\right)$, the acceleration is preferentially oriented in the upstream direction $(\phi= \pm \pi)$, while larger distances to the wall $\left(y^{+}>30\right)$ are associated preferentially with the transverse direction $(\phi= \pm \pi / 2)$. In figure $9(b)$, it is seen that with increasing 


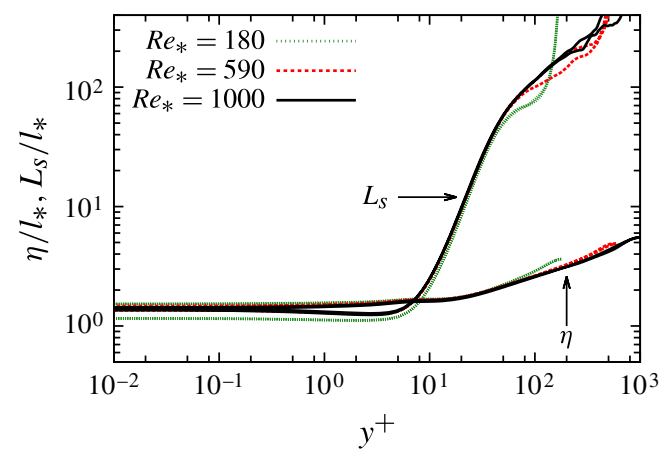

FIGURE 10. (Colour online) Comparison between length scale $L_{s}=\sqrt{\langle\varepsilon\rangle / S^{3}}$ and $\eta=\langle\varepsilon\rangle^{-1 / 4} v^{3 / 4}$ in wall units where $l_{*}=v / u_{*} ; R e_{*}=180,590$ and 1000.

$y^{+}, P(\theta)$ approaches its isotropic distribution $P_{\theta, \text { iso }}(\theta)$ given by (3.20). This figure also displays that close to the wall, the most probable value of $\theta$ is close to 0 , which means that the orientation is almost parallel to the wall. The slightly positively skewed p.d.f. of $\theta$ close to the wall may be attributed to the non-zero positive mean vertical pressure gradient (Pope 2000).

As a condition of local isotropy in the inertial subrange, it was required by Champagne, Harris \& Corsin (1970) to have the time scale of energy transfer much smaller than the mean shear time scale, at which energy is produced: $\left(k^{3} \mathscr{E}(k)\right)^{-1 / 2} \ll$ $1 / S$, where $k$ is the wavenumber, $\mathscr{E}$ is the energy spectra, $S=(1 / 2) \mathrm{d}\langle u\rangle / \mathrm{d} y$ is the mean shear. In terms of length scales, with $\mathscr{E}(k) \propto\langle\varepsilon\rangle^{2 / 3} k^{-5 / 3}$, this condition is $k^{-1} \ll \sqrt{\langle\varepsilon\rangle / S^{3}}$. According to Champagne et al. (1970) $L_{s}=\sqrt{\langle\varepsilon\rangle / S^{3}}$ is the length scale separating the shear-dominated large scales and the region where local isotropy may be encountered. In figure $10, L_{s}$ is compared to the Kolmogorov length scale $\eta=\langle\varepsilon\rangle^{-1 / 4} v^{3 / 4}$ which is a typical scale for the acceleration. Both scales are given in units of the viscous sublayer length scale $l_{*}=v / u_{*}$. It is seen that in the viscous sublayer $\left(y^{+}<5\right), L_{s}$ and $\eta$ are equal to each other, and are very close to the viscous length scale $l_{*}=v / u_{*}$. This implies that, in this region, the flow is strongly anisotropic even at the smallest scale. When distance to the wall increases, $L_{s}$ grows much faster than $\eta$. For example at $y^{+}=50$ we have $L_{s} / \eta \approx 100$, satisfying the condition for local isotropy given by Champagne et al. (1970).

The DNS data in figures 8 and 9 suggest relaxation of orientation of acceleration towards statistical isotropy with increasing distance to the wall: at $y^{+}<10$, the preferential orientation is aligned with the wall, while at $y^{+}=50$, statistical isotropy of orientation is almost reached. Also, from figure 9 it is seen that the mean components of the acceleration orientation are very close to zero. The linear increase of $\left\langle\sin ^{2} \theta\right\rangle$ when $y^{+}$increases to $y^{+} \approx 20$ may imply a diffusive character (or random walk) of the $\theta$-evolution towards statistical isotropy of the acceleration orientation. In order to reproduce such behaviour, the Brownian random walk on a unit sphere, with relaxation towards a uniform isotropic distribution, is introduced in $\S 4$.

Another property of $\phi$ and $\theta$ is that to some extent they may be viewed as two statistically independent angles. This is demonstrated in figure 11 in which for $R e_{*}=1000$, p.d.f.s of $\theta$ conditioned on $\phi$ are presented in the highly anisotropic region, $y^{+}=5$ and $y^{+}=20$. One can see that $\phi$ has very little effect on the distribution of $\theta$. It is also interesting to present the acceleration magnitude as a function of the 

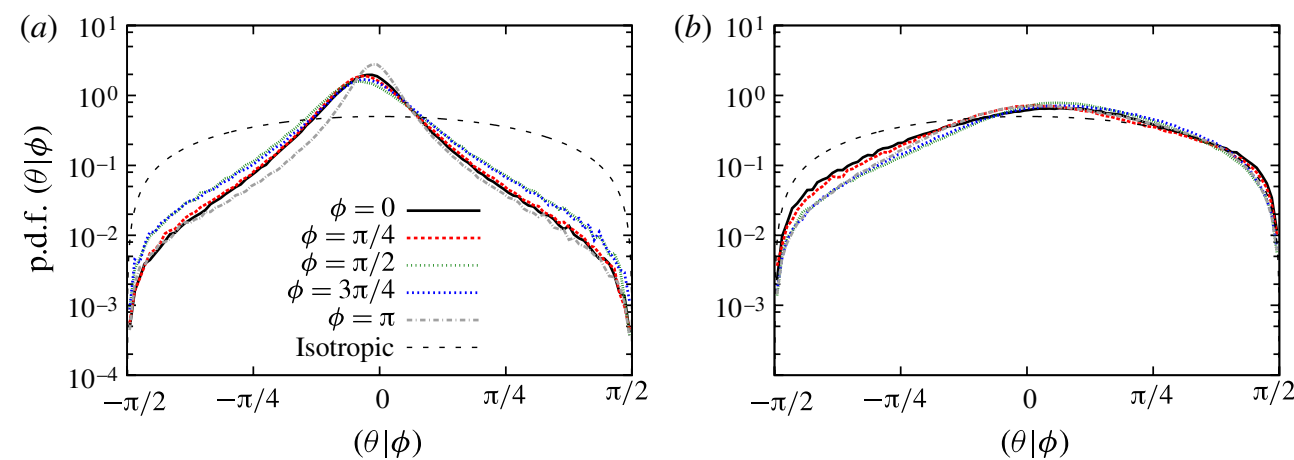

FIGURE 11. (Colour online) p.d.f. of $\theta$ conditioned on $\phi$ for $\operatorname{Re}_{*}=1000$ at $y^{+}=5(a)$ and at $y^{+}=20(b)$. Comparison with the isotropic distribution $P_{\theta, \text { iso }}$ as given by (3.20).

acceleration orientation. The mean norm of acceleration conditionally averaged on its orientation is given in 12 for $R e_{*}=1000$. Figures $12(a)$ and $12(b)$ show the mean profiles conditioned on $\phi$ and $\theta$, respectively. It is clearly seen that in the viscous wall region $\left(y^{+}<30\right)$, the acceleration magnitude depends on its orientation. For example, in the viscous layer, the magnitude of acceleration is dominated by events such as ejections (positive $\theta$ ). Close to the wall (in the viscous sublayer), the acceleration in the upstream direction appears to be also significant, providing the peak around $y^{+} \approx 5$ in the acceleration norm profile conditionally on $\phi= \pm \pi$. However, for larger $y^{+}$, $y^{+}>30$, the preferential orientations are no longer seen. Another illustration comes from figure 13 where p.d.f.s of the acceleration norm conditioned on $\phi$ and $\theta$ at $y^{+}=20$ are shown. Already by $y^{+}=20$ these p.d.f.s are practically independent of the value of orientation angle; they stay log-normal as was already observed for the unconditional p.d.f. in figure 3 . We observe from our DNS that this log-normality persists for greater distances to the wall, and for the other Reynolds numbers.

A stochastic simulation of the subgrid acceleration is provided in the subsequent sections. The proposed models are based on statistical properties obtained by DNS, as described above for $R e_{*}=180,590$ and 1000 .

\section{Stochastic model for acceleration}

In the framework of (1.1), the stochastic model for the acceleration is given by the product of two independent stochastic processes, one for the acceleration modulus, the other for its orientation.

For the acceleration norm we consider that the stochastic process evolves with increasing distance from the wall, and not locally with time, in contrast with Sabel'nikov et al. (2011). Such a process will be characterized by correlation across the whole channel. Two different stochastic processes for the acceleration modulus are proposed hereafter, both in the framework of fragmentation under scaling symmetry. The homogeneous scaling symmetry transformations across the channel are defined by the operator $\exp [\ln a(\partial / \partial \xi) \xi]$ with $\xi=h-y / h$ and $0<\alpha<1$. These homogeneous transformations across the channel induce a transformation group for the acceleration modulus whose parameter is $\tau=\ln (h /(h-y))$. The proposition here is to describe the statistical evolution from the wall to the channel centreline through this parameter only. This parameter, referred to as the evolution parameter, was introduced in 

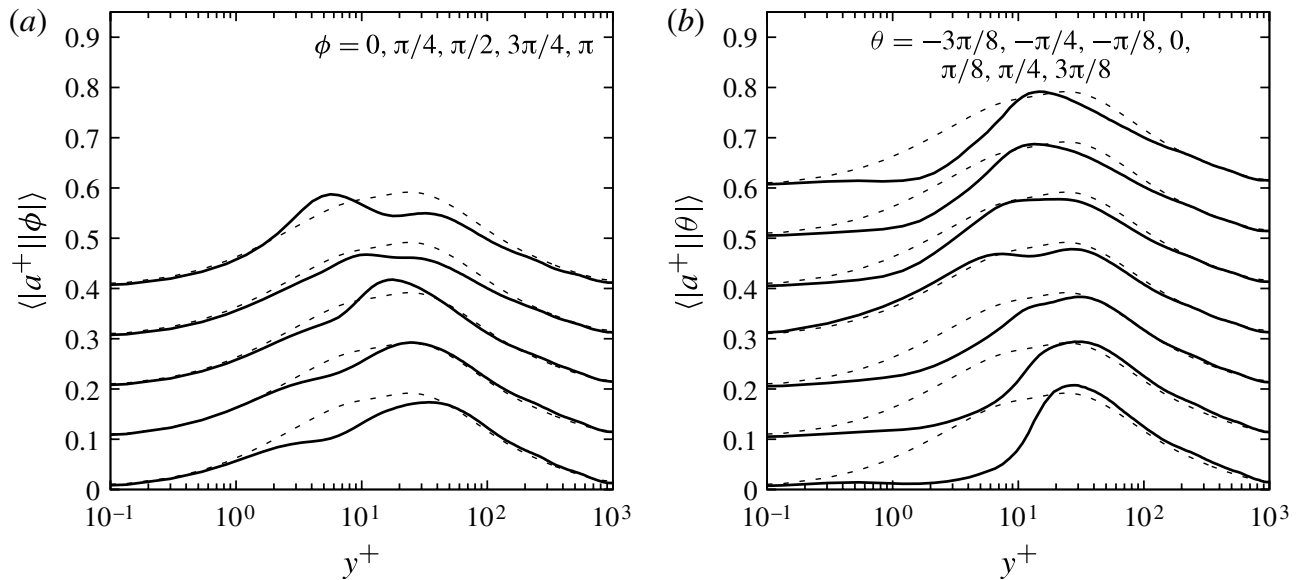

FIGURE 12. Profile of the variance of the acceleration norm conditioned on $\phi(a)$ and $\theta$ (b) for different values of the angle (each shifted upward by 0.1 unit, with the bottom line corresponding to the lowest value of $\phi$ and $\theta$ respectively, from DNS at $\operatorname{Re}_{*}=1000$ (continuous lines), comparison with the unconditioned profile (dashed lines).
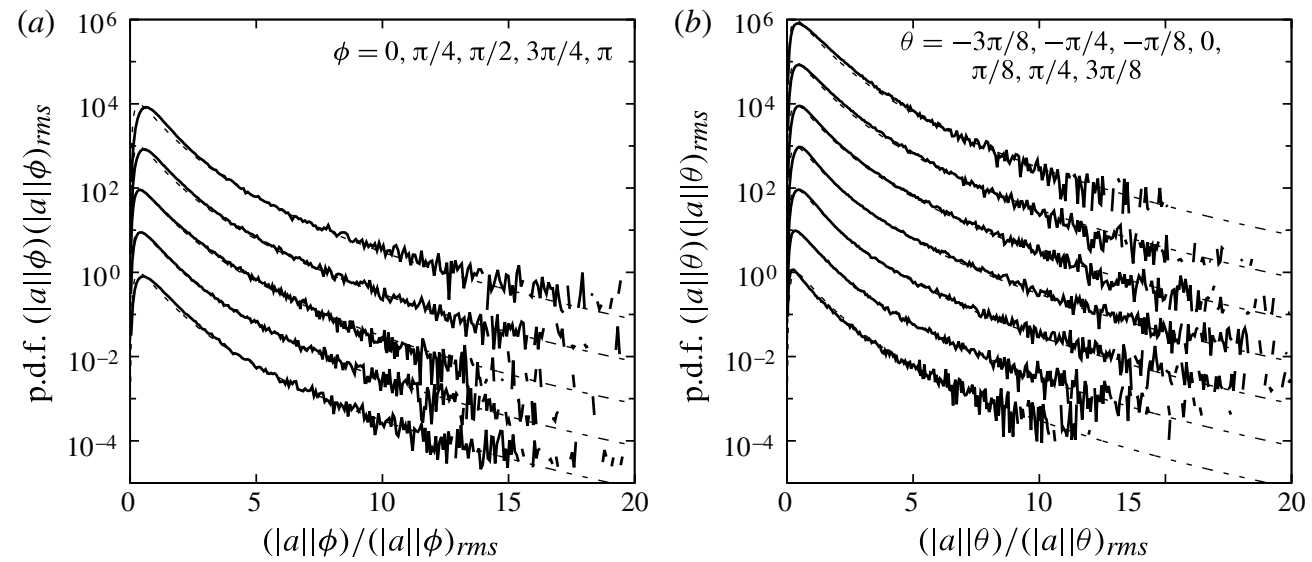

FIGURE 13. p.d.f. of $|a|$ normalized by its standard deviation conditioned on $(a) \phi$ and (b) $\theta$ for $R e_{*}=1000$ at $y^{+}=20$ (each shifted upward by one decade, with the bottom line corresponding to the lowest value of $\phi$ and $\theta$ respectively). Comparison with log-normal distribution with parameters: $\mu=-\sigma^{2} / 2$ and $\sigma^{2}=\ln (2)$ (dashed lines).

the previous section. More arguments on the use of such an evolution parameter in describing the turbulence under continuous symmetry scaling can be found in Saveliev \& Gorokhovski $(2012,2005)$ and Gorokhovski (2003). The expression for $\tau$ is convenient, since at the wall $\tau=0$, and it tends to infinity on the channel centreline.

In terms of Kadanoff (2000), the first model represents a 'non-self-averaging' stochastic process. In this process (Gorokhovski \& Saveliev 2008), the Kolmogorov log-normal distribution represents the earlier asymptotic distribution, with two parameters: the first two logarithmic moments of the fragmentation intensity spectrum. The later asymptotic in this process is a power law with a single parameter, given by 
the ratio of these two logarithmic moments. These moments are introduced here as a function of the logarithm of the Reynolds number. The second model assessed in this paper represents a 'self-averaging' process. Consistently with the DNS, this process starts with a log-normal distribution close to the wall. With increasing distance from the wall, this distribution translates in the sample log-space, in such a way that in the channel mid-plane, its mean is relaxed to assumed estimations from homogeneous isotropic turbulence.

Simultaneously, the orientation of acceleration is stochastically simulated by a random walk on a sphere. The stochastic process for unit vector of orientation $e_{i}(t)$ evolves hereafter with the parameter $\tau$ as well as with time. These two evolutions of the orientation are characterized by a short-scale correlation. In time, the components of the orientation vector $e_{i}\left(e_{k} e_{k}=1\right)$ are assumed to be correlated on the Kolmogorov time scale $\tau_{\eta}$ similarly to Sabel'nikov et al. (2011) for consistency with the Kolmogorov-Oboukhov 41 theory, while with $\tau$ the short-scale correlation will reproduce the evolution towards isotropy observed from the DNS.

\subsection{Two different models for the acceleration modulus}

In our model, the local acceleration norm is expressed as $|a|=y\left(u_{*}^{2} / v\right) f$. Here $y u_{*}^{2} / \nu$ is assumed to be a characteristic velocity increment at the point of interest $y$, and $f$ corresponds to the frequency at which this increment changes. In wall units, the above relation becomes:

$$
\left|a^{+}\right|=y^{+} f^{+},
$$

where $f^{+}=v / u_{*}^{2} f$. It is supposed that $f$ is a stochastic variable evolving across the channel. The following assumptions are made for its evolution. Near the wall, it is characterized mostly by high-frequency events, thereby emulating small-scale turbulent motions. In the outer region, $f$ is characterized by low frequencies, which may be alternated by rare events of high frequency. Those rare events may represent ejections of small-scale turbulence towards the outer region. The main assumption is that with increasing wall distance, the frequency $f$ is governed by a fragmentation process under scaling symmetry, i.e. $f \rightarrow \alpha f$, where $0<\alpha<1$ is a random coefficient (Gorokhovski \& Saveliev 2008). In this process, for any value of $\tau$ in the evolution of the distribution $G(f ; \tau)$, we have $\left\langle(\ln f-\langle\ln f\rangle)^{2}\right\rangle /\langle\ln f\rangle=\left\langle\ln ^{2} \alpha\right\rangle /\langle\ln \alpha\rangle$, with $\langle\ln f\rangle=\int_{0}^{\infty} G(f) \ln f \mathrm{~d} f$. Here $\langle\ln \alpha\rangle$ and $\left\langle\ln ^{2} \alpha\right\rangle$ are the first two logarithmic moments of $\alpha$. In the Ito interpretation, the corresponding stochastic process is:

$$
\mathrm{d} f=\left(\langle\ln \alpha\rangle+\left\langle\ln ^{2} \alpha\right\rangle / 2\right) f \mathrm{~d} \tau+\sqrt{\left\langle\ln ^{2} \alpha\right\rangle / 2} f \mathrm{~d} W(\tau),
$$

where $\mathrm{d} W(\tau)$ is the Wiener process: $\langle\mathrm{d} W(\tau)\rangle=0$ and $\left\langle\mathrm{d} W(\tau)^{2}\right\rangle=2 \mathrm{~d} \tau$. It is prescribed further that $\left\langle\ln ^{2} \alpha\right\rangle /\langle\ln \alpha\rangle=-1 / 2$, and $\langle\ln \alpha\rangle=-k_{1} R e_{*}$, where $k_{1}=0.04$ is determined as the best fit to the DNS data.

In order to start the stochastic process (4.2), one needs to assume the initial distribution of $f$. To this end, the frequency $f$ at the wall $(\tau=0)$, denoted here by $f_{w}$, is given as follows. From (4.1) we have: $f_{w} /\left(u_{*}^{2} / v\right)=\lim _{y \rightarrow 0}\left\langle\left|a^{+}\right|\right\rangle / y^{+}$. Since close to the wall, according to DNS (see figures 6 and 14), the quantity $\langle|a|\rangle / \ln R e_{*}$ is a linear function of $y^{+}$and is independent of the Reynolds number, one may write:

$$
\frac{f_{w}}{u_{*}^{2} / \nu}=k_{2} \ln R e_{*},
$$




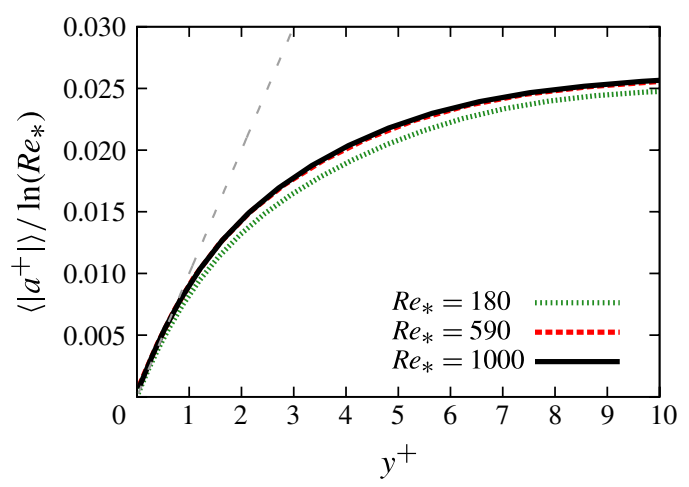

FIgURE 14. (Colour online) Profile of the norm of acceleration for $R e=180,590$ and 1000 from DNS, zoom in the near-wall region. Dashed line: 0.01 slope.
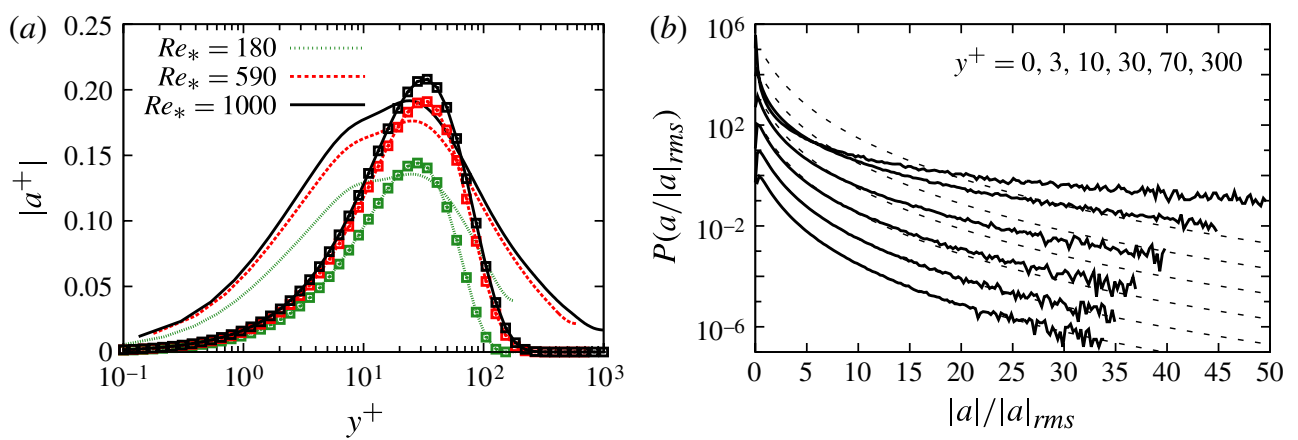

FIGURE 15. (Colour online) (a) Mean value for $|a|$ from the stochastic model (4.2) (symbols) and comparison with DNS (lines) for $R e_{*}=180,590$ and 1000. (b) p.d.f. of $|a|$ from the stochastic model (4.2) for $R e_{*}=1000$ and $y^{+}=0,3,10,30,70$ and 300, equivalent respectively to $\tau=0,3 \times 10^{-3}, 10^{-2}, 3 \times 10^{-2}, 7.2 \times 10^{-2}$ and 0.37 , each shifted upward by one decade, with the bottom line corresponding to the lowest value of $y^{+}$. Comparison with the log-normal distribution of parameters: $\mu=-\sigma^{2} / 2$ and $\sigma^{2}=\ln (2)$ (dashed lines).

where $k_{2}$ is a constant with ascribed value 0.01 from DNS. In figure 14, the profile of $\langle|a|\rangle / \ln R e_{*}$ from the DNS is compared to line $0.01 y^{+}$. Finally, $f / f_{w}$ is chosen to be $\log$-normally distributed at the wall, with the mean equal to variance, i.e. values $-\ln \sqrt{2}$ and $\ln 2$ are ascribed to the mean and variance of $\ln \left(f / f_{w}\right)$, respectively. These assumptions, namely the presumed log-normal distribution of $f / f_{w}$ as well as the equality of the mean and the variance, are consistent with observations from DNS (figures 2 and 3 and (3.1)-(3.4)).

The statistics obtained by (4.1)-(4.3), using $k_{1}=0.04$ and $k_{2}=0.01$, are presented in figure 15. In figure 15(a) the mean profile of the acceleration norm is shown against DNS for $R e_{*}=180,590$ and 1000. It is seen that although the evolution of the profiles is only qualitatively reproduced by our model, the maximum values agree quite well with the DNS data, and the agreement is satisfactory in the range $10<y^{+}<100$. In figure $15(b)$ we present the p.d.f.s of $|a|$ as obtained by the stochastic model at different $\tau$. These distributions are compared with the log-normal distribution, in which the mean is equal to the standard deviation. As prescribed 
by the wall condition, it is seen that for small $\tau$, the p.d.f. is quite close to the $\log$-normal shape. However when $\tau$ increases further, the p.d.f. develops very stretched tails, which may represent rare events of intense acceleration. This process has no characteristic scale and presents a self-similar evolution across the channel. This leads to the development of a power-law distribution for the acceleration norm. However, such an evolution towards the power-law distribution at large values of $\tau$ clearly does not correspond to the DNS observations of turbulent channel flow, where the distribution remains log-normal across the whole channel, and $|a|_{r m s} \approx\langle|a|\rangle$. These observations suggest a cut-off of the fragmentation frequency with relaxation of the p.d.f.s to the log-normal distribution.

This motivates assessing another model. In the second model, the relaxation parameter is imposed by the 'external' turbulent stream. Since in the DNS, $|a|$ is $\log$-normally distributed over the entire channel, and $|a|_{r m s} \approx\langle|a|\rangle$, the same behaviour should be attributed to $f^{+}\left(f^{+}\right.$remains defined by (4.1): $\left.f^{+}=\left|a^{+}\right| / y^{+}\right)$. For a given $\tau$, $\chi=\ln f^{+}$is supposed to be Gaussian, with $f_{r m s}^{+}(\tau)=\left\langle f^{+}\right\rangle(\tau)$. It is helpful to remark that for the following Ornstein-Uhlenbeck process:

$$
\mathrm{d} \chi=-\left(\chi-\mu_{\infty}\right) \frac{\mathrm{d} \tau}{T_{\chi}(\tau)}+\sqrt{\frac{2 \sigma_{\chi}^{2}}{T_{\chi}(\tau)}} \mathrm{d} W(\tau),
$$

the solution for the first and second moments is given respectively by:

$$
\begin{aligned}
\langle\chi\rangle(\tau) & =\mu_{\infty}+\left(\mu_{0}-\mu_{\infty}\right) \exp [-\Gamma(0, \tau)], \\
\left\langle\chi^{\prime 2}\right\rangle(\tau) & =\left.\left\langle\chi^{\prime 2}\right\rangle\right|_{\tau=0} \exp [-2 \Gamma(0, \tau)]+2 \sigma_{\chi}^{2} \int_{0}^{\tau} \exp [-2 \Gamma(s, \tau)] \frac{\mathrm{d} s}{T_{\chi}(s)},
\end{aligned}
$$

where $\Gamma\left(\tau_{1}, \tau_{2}\right)=\int_{\tau_{1}}^{\tau_{2}} \mathrm{~d} s / T_{\chi}(s)$ and $\mu_{\infty}$ and $\sigma_{\chi}^{2}$ are given parameters of (4.4); $T_{\chi}=T_{\chi}(\tau)$ is a given positive function; $\mu_{0}$ and $\left.\left\langle\chi^{12}\right\rangle\right|_{\tau=0}$ represent respectively the mean and the variance at the wall (i.e. $\tau=0)$ and $\mathrm{d} W(\tau)$ is the Wiener process $\left(\langle\mathrm{d} W(\tau)\rangle=0\right.$ and $\left.\left\langle\mathrm{d} W(\tau)^{2}\right\rangle=2 \mathrm{~d} \tau\right)$. On the centreline, $\tau \rightarrow \infty$, from (4.5) we have:

$$
\langle\chi\rangle(\tau \rightarrow \infty)=\left.\langle\ln f\rangle\right|_{\tau \rightarrow \infty}=\mu_{\infty} .
$$

Furthermore from (4.6), if $T_{\chi}=T_{\chi}(\tau)$ is a positive function, and $\left.\left\langle\chi^{\prime 2}\right\rangle\right|_{\tau=0}=\sigma_{\chi}^{2}$, one obtains:

$$
\left\langle\chi^{\prime 2}\right\rangle(\tau)=\left\langle\chi(\tau)^{2}\right\rangle-\langle\chi(\tau)\rangle^{2}=\sigma_{\chi}^{2}
$$

for any $\tau>0$. This implies that once the Gaussian distribution for $\chi(\tau)$ (the $\log$-normal distribution for $f(\tau))$ is assumed at $\tau=0$, the process (4.4) translates this distribution in the sample space with increasing $\tau$. Approaching the channel centreline, its mean tends to (4.7). Here again, from the definition of moments of the log-normal distribution, the case $f_{r m s}^{+}(\tau)=\left\langle f^{+}\right\rangle(\tau)$ corresponds to $\sigma_{\chi}^{2}=\ln 2$. Denoting $f_{c}=\langle f(\tau \rightarrow \infty)\rangle=\exp \left(\mu_{\infty}+\sigma_{\chi}^{2} / 2\right)$, the corresponding stochastic equation for $f^{+}(\tau)=\exp (\chi(\tau))$ may be obtained by using the Ito transformation. Following the log-normal stochastic model of Pope \& Chen (1990) and Pope (1991), this equation is:

$$
\frac{\mathrm{d} f}{f}=-\left(\ln \left(\frac{f}{f_{c}}\right)-\frac{\sigma^{2}}{2}\right) \frac{\mathrm{d} \tau}{T_{\chi}(\tau)}+\sqrt{\frac{2 \sigma_{\chi}^{2}}{T_{\chi}(\tau)}} \mathrm{d} W(\tau) .
$$

Three parameters have to be specified from DNS: the mean quantity $f_{c}=\langle f(\tau \rightarrow$ $\infty)\rangle$ on the centreline; the correlation scale $T_{\chi}(\tau)$ as a function of $\tau$; and the mean 

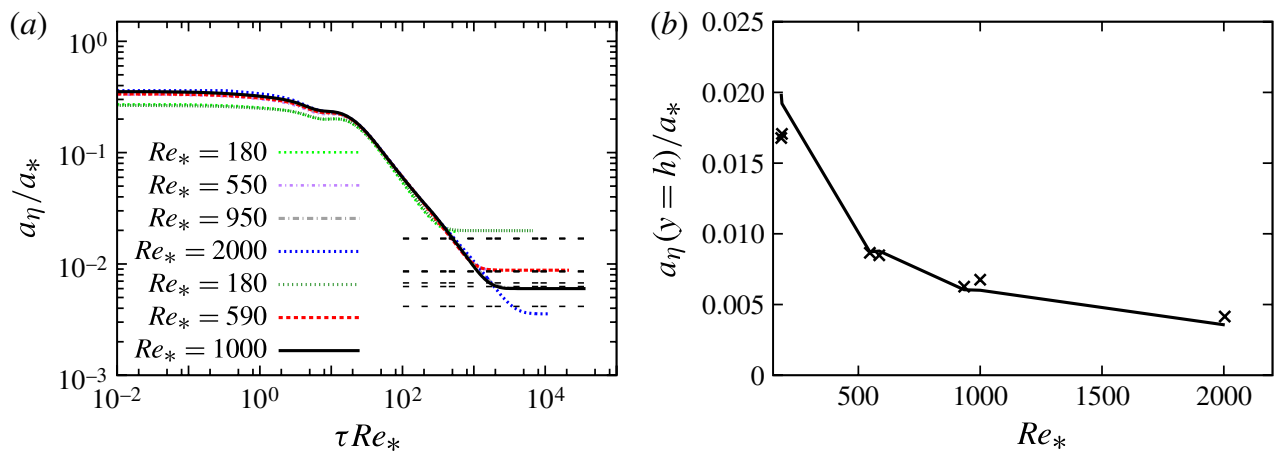

FIgURE 16. (Colour online) (a) Profiles of the Kolmogorov acceleration against $\tau R e_{*}$ obtained by DNS with Reynolds numbers from $R e_{*}=180$ to 2000 (the first four curves, $R e_{*}=180,550,950,2000$, are from DNS of Hoyas \& Jiménez (2008) and the last three are from our DNS), and estimation from (4.11) with $k_{1}=0.12$ (dashed lines). (b) Comparison of the Kolmogorov acceleration at the channel centre from (4.11) with $k_{3}=0.12$ (crosses) and the DNS (continuous lines).

quantity $f_{w}=\langle f(\tau=0)\rangle$. The latter is defined as in the first model (figure 14 and (4.3)). Namely, $f_{w}^{+}=k_{2} \ln R e_{*}$ with $k_{2}=0.01$, and the initial log-normal distribution for $f / f_{w}$ with parameters $\sigma_{x}^{2}=\ln 2$ and $\mu_{0}=-(1 / 2) \sigma_{x}^{2}$ is assumed.

In order to obtain the mean quantity $f_{c}=\langle f\rangle(\tau \rightarrow \infty)$ in the channel mid-plane, we applied estimations used for HIT. By definition, $f_{c}^{+}=\left\langle|a(y=h)|^{+}\right\rangle / R e_{*}$, and according to (3.11):

$$
\frac{\left.\langle|a|\rangle\right|_{y=h}}{\left.a_{\eta}\right|_{y=h}}=\kappa \ln R e_{*} .
$$

Here the Kolmogorov acceleration in the channel mid-plane is given by $\left.a_{\eta}\right|_{y=h}=$ $\varepsilon_{y=h}^{3 / 4} / \nu^{-1 / 4}, \varepsilon_{y=h}$ being the mean dissipation in the mid-plane. Using the Taylor estimation of the dissipation in isotropic turbulence, $\varepsilon \approx 15 v u_{r m s}^{2} / \lambda^{2}$, and using the channel mid-plane height, instead of the integral length scale, in the relationship between the Taylor and integral length scales, $\lambda=h / \sqrt{R e_{c}}$, the dissipation in the mid-plane is estimated as

$$
\varepsilon_{y=h} \approx k_{3} v \frac{u_{r m s}^{2}(y=h)}{h^{2}} R e_{c},
$$

where $k_{3}$ is a constant determined by DNS. In figure 16 the acceleration scale $\left.a_{\eta}\right|_{y=h}=\varepsilon_{y=h}^{3 / 4} / v^{-1 / 4}$ for two cases is compared: the case when the mean dissipation at $y=h$ is given by DNS; and when that dissipation is obtained by (4.11), with $k_{3}=0.12$. The different Reynolds numbers from our DNS and the DNS of Hoyas \& Jiménez (2008) are taken. For a broad range of Reynolds numbers, estimation (4.11) with the chosen $k_{3}=0.12$, predicts quite correctly the magnitude of $\left.a_{\eta}\right|_{y=h}$. Then, (4.10) predicts $\left.\langle|a|\rangle\right|_{y=h}$ and, consequently, $f_{c}$ :

$$
f_{c}^{+}=\kappa \frac{\ln \operatorname{Re}_{*}}{\operatorname{Re}_{*}}\left(\frac{\varepsilon_{y=h}}{\varepsilon_{*}}\right)^{3 / 4} .
$$

The correlation scale $T_{\chi}(\tau)$ is determined from the autocorrelation of the acceleration norm in the wall-normal direction. The autocorrelation coefficient of the 
acceleration norm, for each fixed homogeneous plane parallel to the wall, at height $y$, is defined as:

$$
\rho_{|a|}(y, \boldsymbol{r})=\frac{\left\langle|a|^{\prime}(\boldsymbol{x})|a|^{\prime}(\boldsymbol{x}+\boldsymbol{r})\right\rangle}{\left(\left\langle|a|^{\prime}(\boldsymbol{x})^{2}\right\rangle\left\langle|a|^{\prime}(\boldsymbol{x}+\boldsymbol{r})^{2}\right\rangle\right)^{1 / 2}} .
$$

Here $|a(\boldsymbol{x})|^{\prime}=|a(\boldsymbol{x})|-\langle|a(\boldsymbol{x})|\rangle$ is the fluctuation of the acceleration norm at point $\boldsymbol{x}$. The distance vector $\boldsymbol{r}$ is chosen in the wall-normal direction. Figure 17(a) represents DNS of this autocorrelation for different distances to the wall. Integration of (4.13) over distances $r$ yields the integral length scale for the acceleration norm. It is seen in figure $17(a)$ that due to the flow confinement, two wall-normal directions have to be distinguished, one towards the channel centreline, and another towards the wall:

$$
\begin{aligned}
& L_{|a|, r+}(y)=\int_{0}^{2 h-y} \rho_{|a|}(y, r) \mathrm{d} r, \\
& L_{|a|, r-}(y)=\int_{-y}^{0} \rho_{|a|}(y, r) \mathrm{d} r .
\end{aligned}
$$

In figure $17(b)$, both integral length scales obtained by DNS are shown as functions of $y^{+}$and for three Reynolds numbers. They are compared with two functions: one is proportional to $y^{+}$, another to $\sqrt{y^{+}}$. It is seen that integral length scales from DNS are very weakly related to the Reynolds number. When the distance to the wall becomes significant, the confinement no longer has an influence, and the difference between the two correlation scales, $L_{|a|, r+}(y)$ and $L_{|a|, r-}(y)$, is small. Furthermore, it is seen that for $y^{+}>30, L_{i n t}^{+} \sim \sqrt{y^{+}}$represents a quite correct approximation. When the stochastic variable $f$ is considered, the same behaviour may be prescribed to its correlation scale $T_{f}$. By analogy with Pope \& Chen (1990), it is suggested that $T_{f} \sim T_{\chi}$ and by inverting (3.16), one obtains:

$$
\frac{L_{\text {int }}\left(y^{+}((\tau))\right.}{h} \approx T_{\chi}(\tau)=\frac{c}{\sqrt{R e_{*}}} \sqrt{1-\mathrm{e}^{-\tau}} .
$$

Here $c$ is a constant supposed to be independent of the Reynolds number. The value $c=25$ is ascribed as the best fit to DNS data.

With these specified parameters, the evolution of $\langle f\rangle(\tau)$ corresponding to (4.9) is given by:

$$
\frac{\langle f\rangle(\tau)}{f_{w}}=\left(\frac{f_{c}}{f_{w}}\right)^{1-\exp \Gamma(\tau)}
$$

where

$$
\Gamma(\tau)=\int_{0}^{\tau} \frac{\mathrm{d} \tau^{\prime}}{T_{\chi}\left(\tau^{\prime}\right)}=2 \frac{\sqrt{R e_{*}}}{c} \ln \left(\sqrt{\mathrm{e}^{\tau}}+\sqrt{\mathrm{e}^{\tau}-1}\right) .
$$

The mean acceleration norm obtained from (4.16), (4.17) and (4.1) is plotted in figure 18 against the DNS profiles. For the three tested Reynolds numbers, the model (4.9) produces a mean acceleration norm in good agreement with DNS data. Moreover, with the prescribed value $\sigma_{\chi}^{2}=\ln 2$ and with the chosen initial conditions, we have $f_{r m s}(\tau)=\langle f\rangle(\tau)$ and the log-normal distribution of $f /\langle f\rangle$, with parameters $\mu=-(1 / 2) \sigma^{2}$ and $\sigma^{2}=\ln 2$. This is consistent with our observations from DNS. 

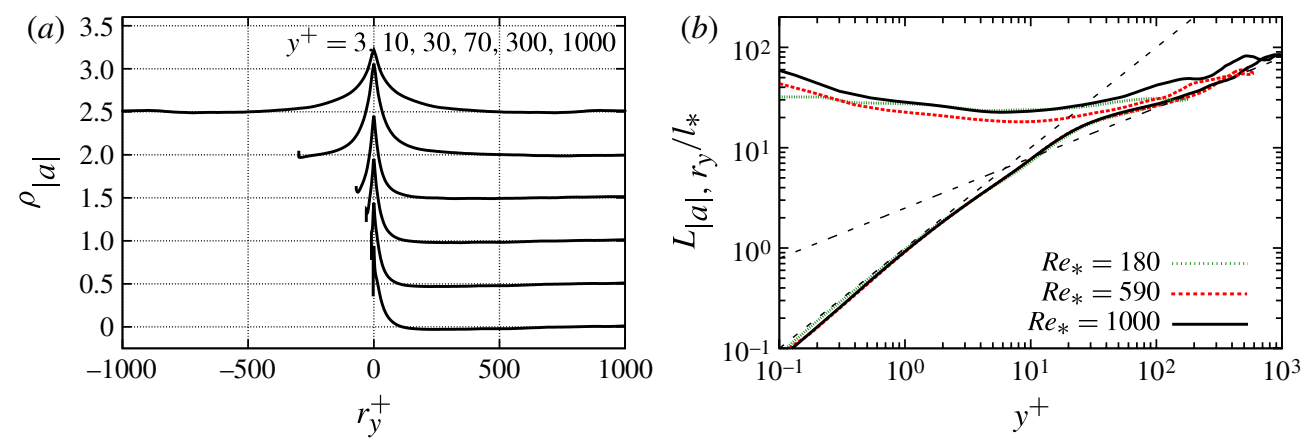

FIGURE 17. (Colour online) (a) Evolution of the autocorrelation coefficient (4.13) in the wall-normal direction obtained by our DNS for $R e_{*}=1000$ at different distances to the wall, each shifted upward by 0.5 units, with the bottom line corresponding to the lowest value of $y^{+}$. (b) Evolution with the wall distance of integral scales $L_{|a|, r+}$ and $L_{|a|, r-}$ (4.14) from DNS for $R e_{*}=180,590$ and 1000. Comparison with $y$ and 2.5 $y^{1 / 2}$ (dashed lines).

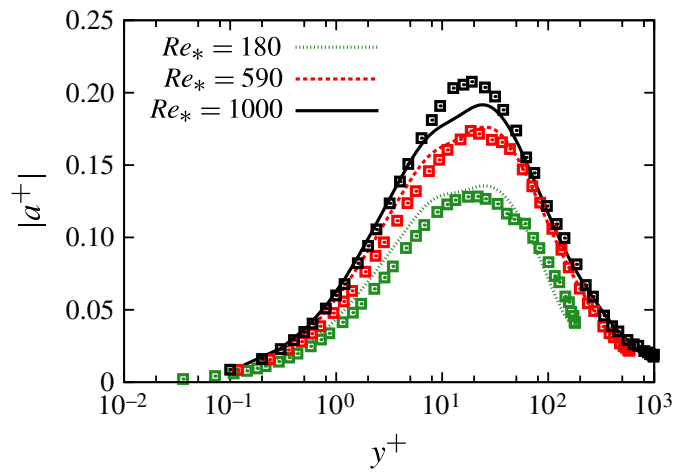

FIgURE 18. (Colour online) Mean value for $|a|$ from the stochastic model (4.9) (symbols) and comparison with DNS (lines) for $R e_{*}=180,590$ and 1000.

\subsection{Model for the orientation of the acceleration vector}

The evolution of the acceleration orientation vector is simulated by a random walk on the surface of the unit sphere. With increasing distance from the wall, the change of position on this sphere is given by the two following expressions:

$$
\gamma=2 D \mathrm{~d} W, \quad 0 \leqslant \beta<2 \pi,
$$

where as illustrated in figure $7, \gamma$ is the arclength between two successive positions on the sphere and $\beta$ is the initial direction from a point $k$ to the point $k+1$. In (4.18), $\beta$ is chosen randomly from a uniform distribution, $D$ is a diffusion coefficient and $\mathrm{d} W$ is the standard Wiener process defined by $\langle\mathrm{d} W\rangle=0,\left\langle\mathrm{~d} W^{2}\right\rangle=2 \mathrm{~d} \tau^{+}$, where $\mathrm{d} \tau^{+}=R e_{*} \mathrm{~d} \tau$ represents the increment in the wall-normal direction. The evolution of the angles $\theta$ and $\phi$ from $\theta_{k}, \phi_{k}$ to $\theta_{k+1}, \phi_{k+1}$, is linked to $\beta$ and $\gamma$ by the following geodesic relations:

$$
\left.\begin{array}{l}
\theta_{k+1}=\sin ^{-1}\left(\sin \theta_{k} \cos \gamma+\cos \theta_{k} \sin \gamma \cos \beta\right), \\
\phi_{k+1}=\phi_{k}+\arg (\zeta),
\end{array}\right\}
$$


where $\zeta$ is a complex number with real and imaginary parts given by $\operatorname{Re}(\zeta)=$ $\sin \beta \sin \gamma \cos \theta_{k}$ and $\operatorname{Im}(\zeta)=\cos \gamma-\sin \theta_{k} \sin \theta_{k+1}$, respectively.

Owing to the finite surface of the sphere, regardless of the initial distribution of $\theta$ and $\phi$, this process reaches a diffusive 'equilibrium' for large $\tau^{+}$. It corresponds to the statistical isotropy of the orientation; then the p.d.f.s of $\theta$ and $\phi$ become:

$$
\left.\begin{array}{l}
P_{\theta}\left(\theta, \tau^{+} \rightarrow \infty\right) \rightarrow P_{\theta, i s o}(\theta), \\
P_{\phi}\left(\phi, \tau^{+} \rightarrow \infty\right) \rightarrow P_{\phi, i s o}(\phi),
\end{array}\right\}
$$

where $P_{\theta, \text { iso }}(\theta)$ and $P_{\phi, i s o}(\phi)$ are given by (3.19) and (3.20), respectively.

Following our DNS observations (figures 8 and 9), the orientation vector is chosen to be initially given by a random orientation in the wall-parallel plane:

$$
\left.\begin{array}{l}
P_{\theta}\left(\theta, \tau^{+}=0\right)=\delta(\theta), \\
P_{\phi}\left(\phi, \tau^{+}=0\right)=P_{\phi, i s o}(\phi),
\end{array}\right\}
$$

where $\delta$ is the Dirac distribution. As seen from the definitions of $\theta$ and $\phi$ (3.18), this condition at the wall gives for the mean orientation vector components: $\left\langle e_{x}(\tau=0)\right\rangle=\left\langle e_{y}(\tau=0)\right\rangle=\left\langle e_{z}(\tau=0)\right\rangle=0$.

In summary, starting with (4.21), and with increasing $\tau^{+}$, the stochastic process given by (4.18) and (4.19) provides a convergence of the p.d.f.s of $\theta$ and $\phi$ towards (4.20). It is worth noting that the statistical azimuthal symmetry (i.e. the invariance of the statistics with an arbitrary rotation in the wall-parallel plane), imposed by the condition at the wall, is preserved during the evolution of the process: $P_{\phi}\left(\phi, \tau^{+}\right)=P_{\phi, i s o}(\phi)$ for all values of $\tau^{+}$. Furthermore, the statistical symmetry of reflection about the 'equator' plane of the sphere $(\theta=0)$ is conserved when $\tau^{+}$ increases. Hence with the condition at the wall (4.21) the ensemble average of the orientation vector components is:

$$
\left\langle e_{x}\right\rangle=\left\langle e_{y}\right\rangle=\left\langle e_{z}\right\rangle=0,
$$

whatever the wall distance.

It is required to provide the Kolmogorov time scale as the correlation time in simulation of the temporal evolution of $e_{i}(t)$. Introducing a correlation of the order of $\tau_{\eta}$ in addition to the wall-normal evolution requires solving a partial differential stochastic equation. As in Sabel'nikov et al. (2011), the simplified procedure is employed here: the new value $e_{i}$ is generated by (4.18) and (4.19) after a time lag $t-t_{0}$ equal to the local Kolmogorov time scale, thereby replacing the exponential relaxation function $\left\langle e_{i}(t) e_{j}\left(t_{0}\right)\right\rangle=\delta_{i j} \exp \left(-2\left|t-t_{0}\right| / \tau_{\eta}\right)$ by its approximation, as a linear function. Similar approximations have been used by Chevillard \& Meneveau (2006) and Afonso \& Meneveau (2010) for modelling the correlation of the velocity gradient deformation.

In (4.18) and (4.19), the only parameter is the diffusion coefficient $D$. Previously, in figure 8 , it has been shown by DNS that the variance of $\theta$ evolves linearly with $y^{+}$in the near-wall region (note that in this region $\tau^{+} \approx y^{+}$). This allows us to estimate the diffusion coefficient $D$. As seen in figure 19, the DNS data suggest that $D=0.01$ is a good approximation for all three tested Reynolds numbers. Using this value, figure 19 shows that model (4.18), (4.19) and (4.21) can correctly predict the DNS profiles of the variance of $\theta$ by giving an evolution towards the isotropic variance.

Another comparison with DNS at $R e_{*}=1000$ is given in figure 20 for the p.d.f.s of $\theta$ at different channel heights $y^{+}$. Here, the comparison is less satisfactory, since 


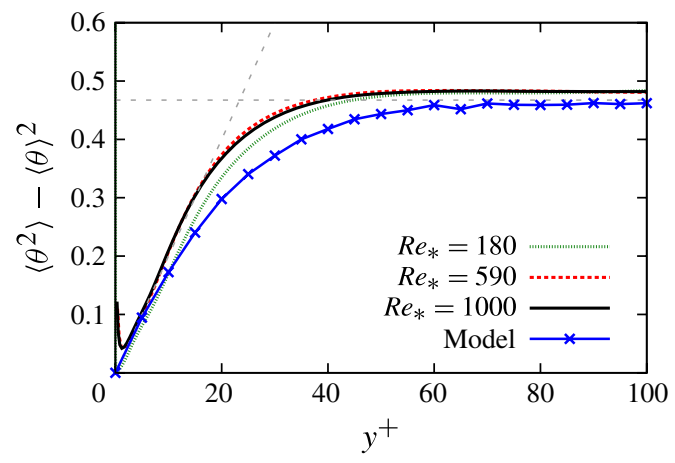

FIGURE 19. (Colour online) Variance of $\theta$. Comparison between DNS $\left(R e_{*}=180,590\right.$ and 1000) and the stochastic model (4.18), (4.19) and (4.21). The horizontal dashed line corresponds to the variance of the isotropic distribution (3.22) and the sloping dashed line to $D y$, with $D=0.01$.

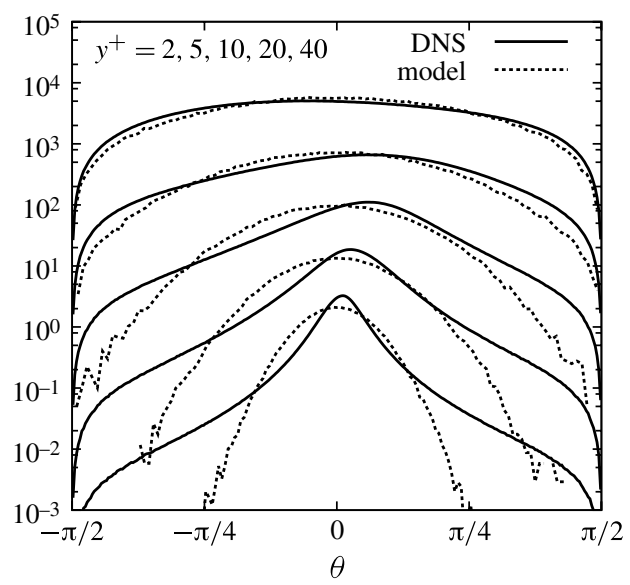

FIGURE 20. p.d.f. of $\theta$ at different distances to the wall, shifted upward by one decade with increasing wall distance. Comparison between the stochastic model (4.18), (4.19) and (4.21) and DNS for $R e_{*}=1000$.

at small wall distances, the Brownian motion on the sphere fails to reproduce the large fluctuations of orientation observed by DNS. Owing to the prescribed distribution at the wall, neither can the small skewness be reproduced. Nevertheless, as expected, with increasing wall distance, the simulated distribution of $\theta$ approaches the isotropic distribution and the DNS distribution.

\section{Large-eddy simulation with stochastic subgrid modelling of acceleration}

In this part of the paper, the stochastic models developed in the previous section are applied, as a subgrid acceleration model, in LES of fully developed turbulent channel flow. This is done in the framework of the LES-SSAM approach (largeeddy simulation coupled with stochastic subgrid acceleration model). The LES-SSAM approach was recently introduced by Sabel'nikov et al. (2011), and applied to HIT 
simulations. In this approach, the instantaneous total acceleration is decomposed into two parts, filtered and residual. Both parts need to be modelled to obtain a closed system of equations. A surrogate flow, simulated by the stochastic Navier-Stokes equations (SNSE), is then considered as an approximation to the instantaneous nonfiltered velocity field. The SNSE derived by Sabel'nikov et al. (2011) is:

$$
\hat{a}_{i}=\frac{\partial \hat{u}_{i}}{\partial t}+\hat{u}_{j} \frac{\partial \hat{u}_{i}}{\partial x_{j}}=-\frac{1}{\rho} \frac{\partial \hat{P}}{\partial x_{i}}+\frac{\partial}{\partial x_{j}}\left[\left(v+v_{t}\right)\left(\frac{\partial \hat{u}_{i}}{\partial x_{j}}+\frac{\partial \hat{u}_{j}}{\partial x_{i}}\right)\right]+a_{i}^{\star} .
$$

Here $\hat{u}_{i}$ represent the components of the surrogate velocity field, $v_{t}$ is an eddy-viscosity model, and $a_{i}^{\star}$ is the stochastic variable. The latter can be thought of as the total acceleration in the non-resolved part of the velocity field which is required to be solenoidal. Finally, $\hat{P}$ is the pressure, that ensures incompressibility of the surrogate velocity field:

$$
\frac{\partial \hat{u}_{j}}{\partial x_{j}}=0
$$

Two models for the stochastic acceleration $a_{i}^{\star}$ are applied to (5.1)-(5.2). Both models are based on the main assumption $a_{i}^{\star}=|a| e_{i}$, along with the use of (4.18)-(4.21) for the modelling of the orientation. In the first model the acceleration norm is simulated by (4.2), whereas in the second model the acceleration norm is given by (4.9). Hereafter, they are referred to as LES-SSAM1 and LES-SSAM2, respectively.

Usually, new terms corresponding to SGS motions are introduced into LES as the divergence of a tensor, unlike the acceleration term in (5.1). This may raise questions concerning momentum conservation in the flow. It follows from (5.1) that:

$$
\oint_{\partial D}\left(+\hat{P} \delta_{i j}-\hat{\tau}_{i j}+\rho \hat{u}_{i} \hat{u}_{j}\right) n_{j} \mathrm{~d} s=\int_{D} \rho a_{i}^{\star} \mathrm{d} v
$$

where $\hat{\tau}_{i j}$ is the viscous stress in the surrogate field, $D$ is an arbitrary domain with surface boundary $\partial D$ and $n_{j}$ is the normal to the surface element $\mathrm{d} s$. Both LES-SSAM1 and LES-SSAM2 give $\left\langle a_{i}^{\star}\right\rangle=0$ for $i=1,2,3$ at every point of the flow. Here, the mean acceleration model is zero because it is given by the product of two independent terms, one for the norm another for the orientation (assumption (1.1)), and because the latter is zero on average, (4.22).

Then on average LES-SSAM1 and LES-SSAM2 are both conservative:

$$
\int_{D} \rho\left\langle a_{i}^{\star}\right\rangle \mathrm{d} v=0 .
$$

Furthermore, the subgrid accelerations are non-correlated in the homogeneous directions ( $x$ and $z$ ); then regarding the right-hand side integral in (5.3) as an ensemble average, one may consider this integral to be approximately zero at each time. This implies a negligible contribution of subgrid scales over a control volume much larger than the mesh cell.

In the rest of this section both LES-SSAM-1 and LES-SSAM-2 are assessed by comparison with the DNS. Further, to emphasize the effect of $a_{i}^{\star}$, this assessment is compared with standard LES using the same mesh and parameters. Note that for this comparison, the DNS and LES-SSAM have not been preliminarily filtered. 


\subsection{Numerical simulations}

The comparisons between LES-SSAM, LES and DNS are made for $R e_{*}=590,1000$ and 2000. Two different mesh resolutions have been used for $R e_{*}=1000$ and 2000. The parameters of the simulations are summarized in table 2 . We note again that in both LES and LES-SSAM identical sets of parameters and the same mesh are used. It should be noted also that the resolution applied in our computation is quite coarse, even for LES, especially for $R e_{*}=2000$. Using a pseudo-spectral method enables us to minimize discretization errors and numerical dissipation effects. Therefore, the computational code used for DNS (see § 2) was used for LES and LES-SSAM, with the following modifications. The subgrid-stress model term $2 v_{t} S_{i j}$ and the acceleration model $a_{i}^{\star}$ are computed as the nonlinear terms. For the turbulent viscosity $v_{t}$ we used the Smagorinsky model with the improvement proposed by Lévêque et al. (2007). This allows us to account for inhomogeneity in the shear flow, without introducing $a d$ hoc wall damping functions (Pope 2000), or the Germano-Lilly dynamical procedure (Germano et al. 1991; Lilly 1992). The shear-improved Smagorinsky model is expressed in the following way (Lévêque et al. 2007):

$$
v_{t}=\ell^{2}(|\bar{S}|-|\langle\bar{S}\rangle|),
$$

where the characteristic strain rate $|\bar{S}|$ is based either on the filtered velocity field in LES, or on the surrogate velocity field in LES-SSAM. $\langle\bar{S}\rangle$ denotes its average over time and in the homogeneous planes, and $\ell$ is a characteristic length scale, $\ell=C_{s} \Delta$ with $\Delta=\left(\Delta_{x} \Delta_{y} \Delta_{z}\right)^{1 / 3}$ and $C_{s}=0.16$. Following Lévêque et al. (2007) $v_{t}$ is required to be greater than $-v$ by clipping lower values. This ensures that the total viscosity $\left(v_{t}+v\right)$ remains positive, thereby avoiding numerical instability. The computations were also performed using two other eddy-viscosity models: the 'classical' Smagorinsky model with a wall damping function and the dynamic Smagorinsky model. With these three turbulent viscosity models the results of the comparisons are very similar, thus we only present the comparison of the simulations with the shear-improved Smagorinsky model of Lévêque et al. (2007).

\subsection{Assessment of LES-SSAM}

In this section comparison between LES-SSAM, LES and DNS is given for the velocity, energy budget and acceleration statistics. Comparisons between the LES and the LES-SSAM aim to emphasize the effect of the stochastic modelling for the acceleration, while the DNS is used as a reference on which LES-SSAM should ideally converge.

First, we focus on the computed value of the Reynolds number, $R e_{c}$, related to the mean velocity in the center of the channel. From table 2, it is seen that both LES-SSAM-1 and LES-SSAM-2, give $R e_{c}$ closer to the DNS, than in the case of the standard LES. The mean velocity in the center of the channel, and consequently, the Reynolds number $R e_{c}$ are often overestimated in LES in comparison to DNS (Germano et al. 1991; Sagaut, Montreuil \& Labbé 1999; Sarghini, Piomelli \& Balaras 1999; Hughes, Oberai \& Mazzei 2001; Volker, Moser \& Venugopal 2002; Park \& Mahesh 2008; Brasseur \& Wei 2010). This overestimation stems from poor spatial resolution of momentum transfer across the near-wall region. In our computation, the mean pressure gradient $\left(R e_{*}=u_{*} h / v=\sqrt{-(1 / \rho)\langle\mathrm{d} p / \mathrm{d} x\rangle} h / v\right)$ is imposed and the mass flow rate adjusts itself in order to produce the prescribed loss of pressure. Thus, the better estimation of the centreline velocity by the LES-SSAM corresponds to an enhanced momentum transfer towards the wall. Does it improve significantly the 


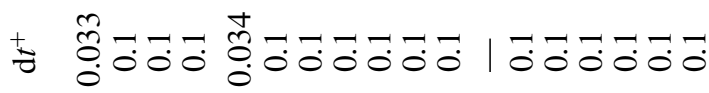

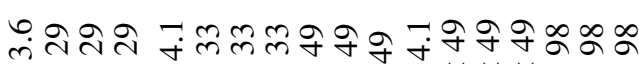
I $\times \times \times \times \times \times \times \times \times \times \times \times \times \times \times \times \times \times$

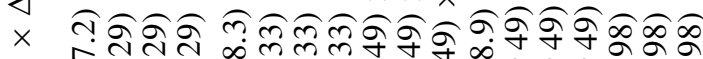
$+2\} 2\} 2\} 2\} 2\} 2\} ? 2\} 2\} 2$

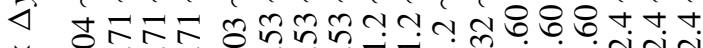

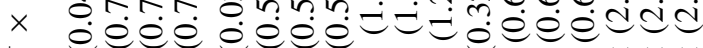
$+\quad e_{x} x_{x} x_{x} \times x \times x_{x} \times x \times x$

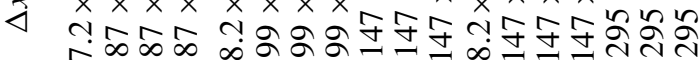

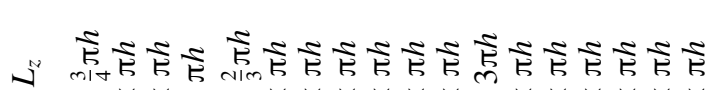
$x \times \times \times \times \times \times \times \times \times \times \times \times \times \times \times \times \times$ ง $\times \times \times \times \times \times \times \times \times \times \times \times \times \times \times \times \times \times \times$

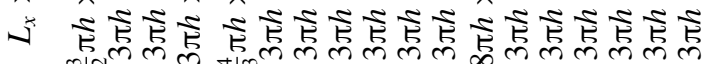

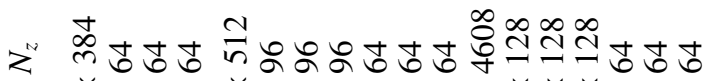

$\times \times \times \times \times \times \times \times \times \times \times \times \times \times \times \times \times \times \times$

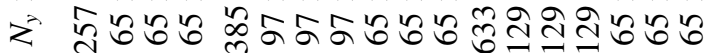
$\times \quad \times \times \times \times \times \times \times \times \times \times \times \times \times \times \times \times \times x$

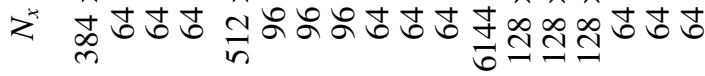

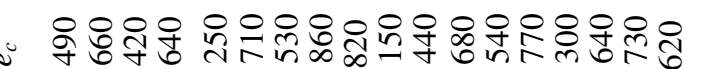

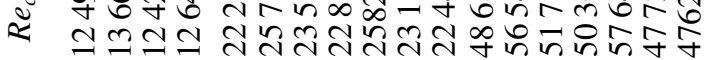

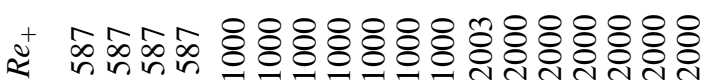

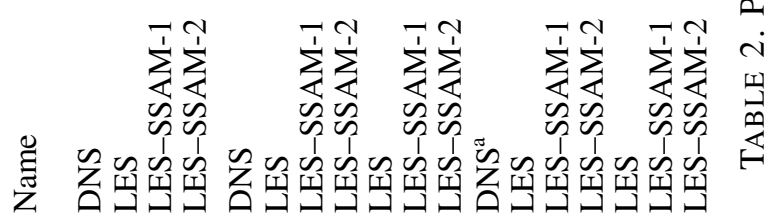


prediction of velocity and acceleration statistics across the whole channel compared to the DNS data? This is the question raised in this Section. The comparison between LES and LES-SSAM is shown here for the coarse resolution. The computation was also carried out for a finer resolution (see table 2), but as expected, this leads to reduced differences between LES-SSAM and standard LES.

In figure 21(a), the mean velocity profiles, corresponding to $R e_{*}=590,1000$ and 2000 obtained by LES-SSAM, by LES, by our DNS, and by the DNS of Hoyas \& Jiménez (2008) are shown. It is seen that the profiles from DNS are better predicted by LES-SSAM than by standard LES. Moreover, the differences between the LES-SSAM-1 and the LES-SSAM-2 are very small.

For the same three Reynolds numbers, the r.m.s. of the three components of velocity is shown in figure $21(b-d)$. In comparison to the standard LES, one can readily see that the predictions of the profiles from the LES-SSAM become better, although the match with the DNS profiles is still not perfect. More specifically, in the near-wall region, when LES-SSAM is employed, the position of the peak, its magnitude, as well as the growth rate of the profiles are closer to the DNS. This is especially visible for the longitudinal component. At the same time, for $R e_{*}=2000$, the r.m.s. values near the centre of the channel are slightly underestimated for both LES-SSAM and LES. It is also seen that for $R e_{*}=590$ and 2000, LES-SSAM-2 provides better prediction than LES-SSAM-1 for the velocity variance of the spanwise component.

In figure 22 , the evolutions of viscous and turbulent stresses, $\tau_{v i s c}=-\rho v\langle\partial u / \partial y\rangle$ and $\tau_{\text {turb }}=-\rho\left\langle u^{\prime} v^{\prime}\right\rangle$, for LES, LES-SSAM and DNS are presented. These stresses are normalized by the total stress $\tau_{\text {tot }}=\tau_{\text {visc }}+\tau_{\text {turb }}$, and they are compared for $R e_{*}=1000$. It is seen that for LES-SSAM, the profiles of the stresses become significantly closer to DNS, in comparison with standard LES. This reflects the fact that the momentum transfer in the wall-normal direction is increased with LES-SSAM. On one hand, the region directly influenced by the wall (the viscous sublayer and the buffer zone) is better predicted. This is clearly visible from the r.m.s. profiles (figure $21 b-d$ ). On the other hand, the mean velocity is improved as seen in figure $21(a)$.

An advantage of LES-SSAM in matching the DNS data can also be seen by comparison of velocity spectra. The one-dimensional spectra for the three velocity components obtained by LES-SSAM, standard LES and DNS are shown in figure 23 for $R e_{*}=1000$, and for two heights in the near-wall region, $y^{+}=5$ and 20 . With the available mesh resolution, the energy of high wavenumbers obtained from LES-SSAM is increased towards the DNS values. This emphasizes that adding a term for modelling the residual acceleration enables a significant increase of the energy contained in the large wavenumbers.

From (5.1) it can be seen that the LES-SSAM approach introduces an additional term for the turbulent kinetic energy balance:

$$
\frac{1}{2} \frac{\mathrm{d}\left\langle u_{i} u_{j}\right\rangle}{\mathrm{d} t}=\left\langle u_{i}^{\prime} a_{i}^{\mathrm{LES}}\right\rangle+\left\langle u_{i}^{\prime} a_{i}^{\star}\right\rangle .
$$

The last term on the right-hand side, $\mathscr{P}_{a}=\left\langle u_{i}^{\prime} a_{i}^{\star}\right\rangle$, represents the turbulent kinetic energy production due to the subgrid stochastic model. In figure 24 this additional term from LES-SSAM-1 and LES-SSAM-2 is compared to the production term: $\mathscr{P}=-\left\langle u^{\prime} v^{\prime}\right\rangle \mathrm{d}\langle u\rangle / \mathrm{d} y$ from DNS. Although the production due to the stochastic model is not expected to have the same behaviour as the production due to the interaction with the mean shear, the comparison between these two terms enables representation of the contribution from the model in the kinetic energy budget. It is seen that although for both models the injection of turbulent kinetic energy by the SGS model 

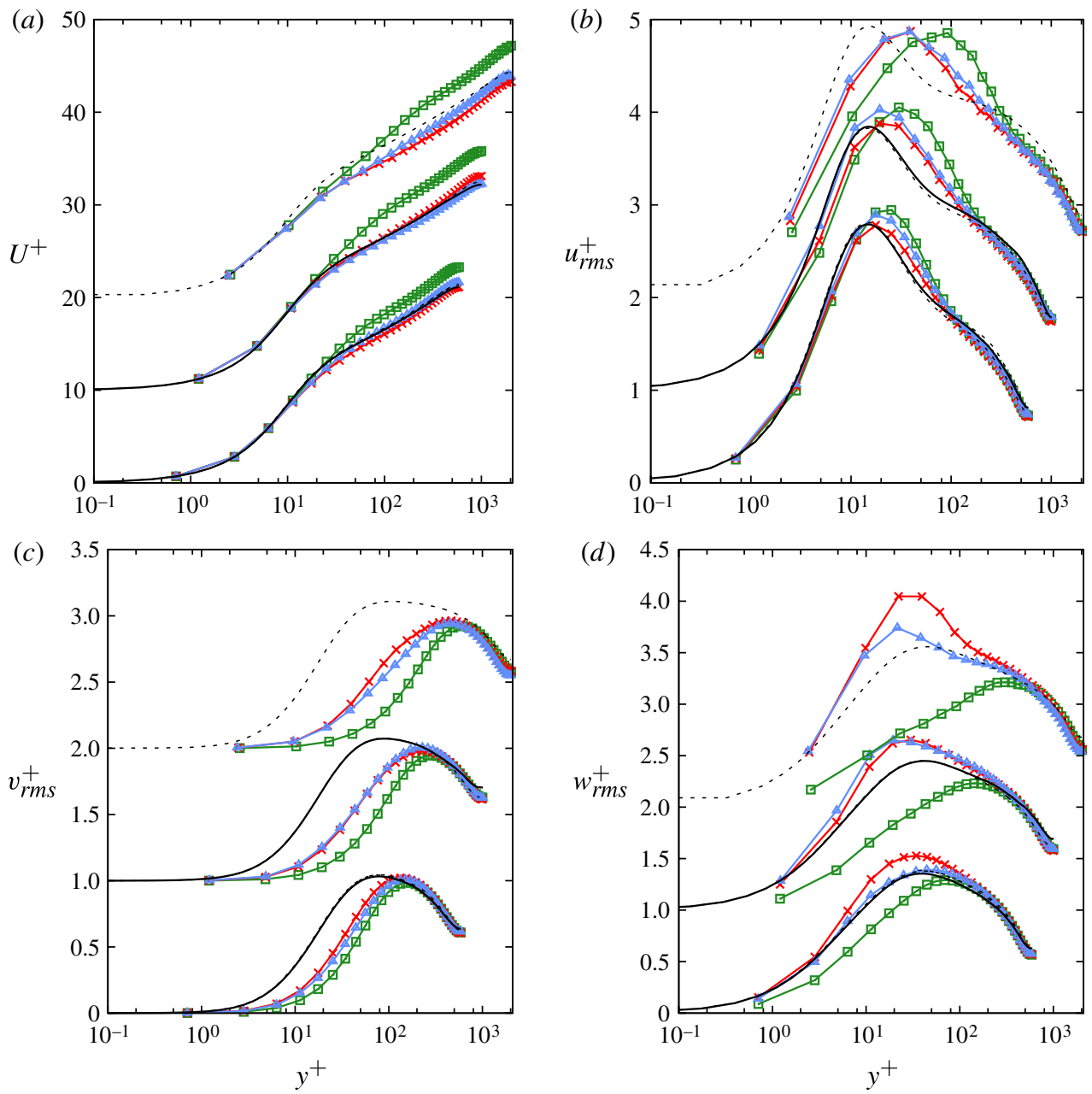

FIGURE 21. (Colour online) Velocity profile for $R e_{*}=590,1000$ and 2000, respectively from bottom to top. Squares: LES; crosses: LES-SSAM-1; triangles: LES-SSAM-2; solid line: our DNS; dashed line: DNS of Hoyas \& Jiménez (2008) for $R_{*}=2000$. (a) Mean velocity (log-linear scales). r.m.s. (log-linear scales) for the streamwise $(b)$, the wall-normal (c) and the spanwise $(d)$ velocity components.

represents only a small part of $\mathscr{P}$ from DNS, this contribution is not negligible. For LES-SSAM-1, shortly after the buffer zone, the subgrid source of energy drops very rapidly, while for LES-SSAM-2 it decreases similarly to DNS.

Figure 25 presents the comparison between LES-SSAM, DNS and LES for the effective rate of kinetic energy production and for the effective rate of dissipation, $\mathscr{P}_{\text {eff }}$ and $\varepsilon_{\text {eff }}$, respectively. The effective dissipation is defined as $\varepsilon_{\text {eff }}=2\left(v+v_{\text {turb }}\right) S_{i j} S_{i j}$, $v_{\text {turb }}=0$ for the DNS. For the LES-SSAM the effective production is $\mathscr{P}_{\text {eff }}=\mathscr{P}+\mathscr{P}_{a}$. First, the turbulent kinetic energy production is much higher with LES-SSAM than with the LES. However, even with the LES-SSAM-2 the production is still largely underestimated compared to the DNS. Adding the acceleration model increases the kinetic energy production directly by $\mathscr{P}_{a}$, and indirectly by a better estimation of the turbulent stress (as observed in figure 22). Note also that the rate of turbulent 


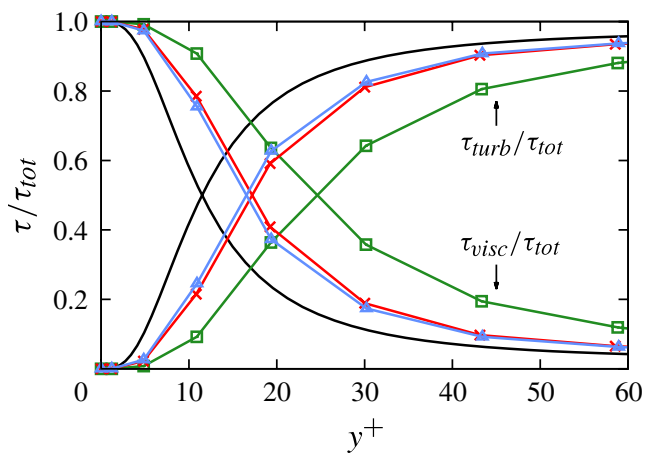

FIGURE 22. (Colour online) Ratios of turbulent $\tau_{\text {turb }}=-\rho\left\langle u^{\prime} v^{\prime}\right\rangle$ and viscous $\tau_{\text {visc }}=$ $-\rho v\langle\partial u / \partial y\rangle$ stresses compared to the total stress $\tau_{\text {tot }}=\tau_{\text {visc }}+\tau_{\text {turb }}$ for $R e_{+}=1000$ in the near-wall region $\left(y^{+}<60\right)$. Squares: LES; crosses: LES-SSAM-1; triangles: LES-SSAM-2; line: DNS.
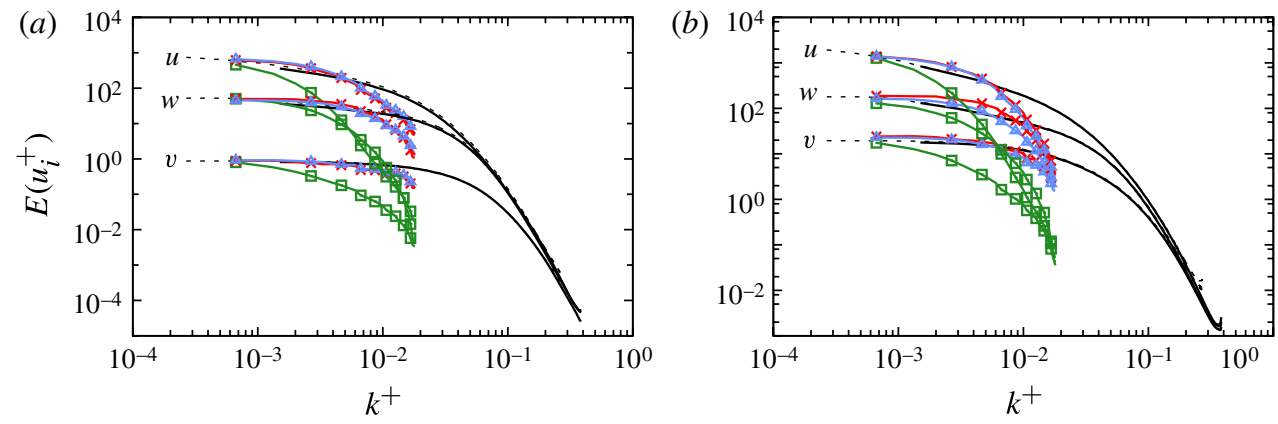

FIGURE 23. (Colour online) Normalized longitudinal one-dimensional spectra of velocity for two distances to the wall: $(a) y^{+}=5$ and $(b) 20$, for $R e_{+}=1000$. Squares: LES; crosses: LES-SSAM-1; triangles: LES-SSAM-2; solid line: DNS; dashed line: DNS of Hoyas \& Jiménez (2008).

kinetic energy dissipation is closer to the DNS in the wall region. This is due to the enhancement of the velocity gradient in LES-SSAM in comparison with standard LES.

The next three figures (figures 26, 27 and 28) show a comparison between the p.d.f.s of acceleration components obtained from LES-SSAM, LES and DNS. These p.d.f.s are presented for $R e_{*}=1000$, and for three wall distances $\left(y^{+}=5,20\right.$ and 950). As is observed from DNS in $\S 3$, the acceleration distribution departs significantly from the normal distribution. This is manifested by the stretched tails, as a result of intermittency on small scales. The p.d.f.s of acceleration obtained by LES-SSAM also present highly stretched tails. Moreover, the computation based on LES-SSAM-1 is characterized by stronger tails of the acceleration distribution than in the case of LES-SSAM-2. This is a direct consequence of rare events given by (4.2). From these figures, it is difficult to show that one formulation of LES-SSAM has an explicit advantage over the other. In the longitudinal acceleration distribution close to the wall $\left(y^{+}=5,20\right)$, the tails are overextended in LES-SSAM-1. Thereby, LES-SSAM-2 has the advantage of being closer to the DNS. However, 


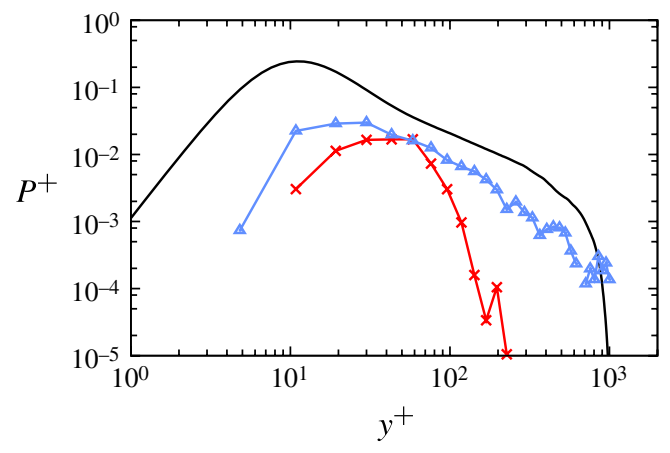

FIgURE 24. (Colour online) Profiles of the additional production term, $\mathscr{P}_{a}=\left\langle u_{i}^{\prime} a_{i}^{\star}\right\rangle$, for $R e_{*}=1000$. Crosses: $\mathscr{P}_{a}$ for LES-SSAM-1; triangles: $\mathscr{P}_{a}$ for LES-SSAM-2; line: $\mathscr{P}=-\left\langle u^{\prime} v^{\prime}\right\rangle \mathrm{d}\langle u\rangle / \mathrm{d} y$ computed by DNS.

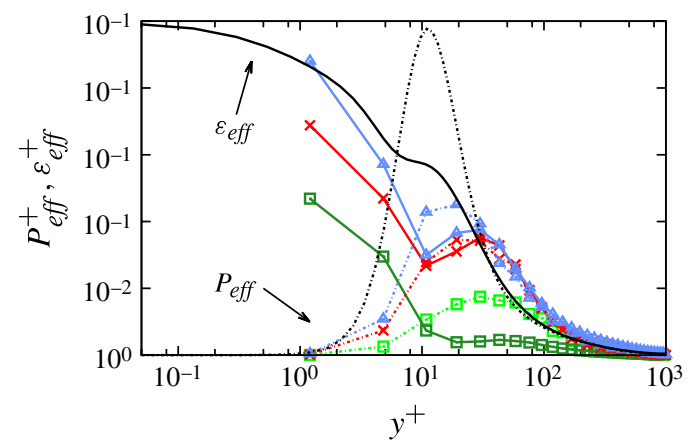

FIGURE 25. (Colour online) Turbulent kinetic energy production and turbulent kinetic energy dissipation for $R e_{*}=1000$. Dashed lines: $\mathscr{P}_{\text {eff }}$; continuous lines: $\varepsilon_{\text {eff }}$. Squares: LES; crosses: LES-SSAM-1; triangles: LES-SSAM-2; line: DNS.

for $y^{+}=950$, LES-SSAM- 1 matches DNS data better than LES-SSAM-2. For the normal component of acceleration, DNS is better predicted by LES-SSAM-1. Simultaneously, the acceleration p.d.f. in the spanwise direction presents overstretched tails in LES-SSAM-1 and then LES-SSAM-2 has an advantage. However, despite these differences, it is clearly seen that both LES-SSAM models are able to capture intermittency effects.

This may be concluded also from the evolution of the velocity increment flatness with separation scale, which is an efficient measure of the intermittency (Frisch 1995). First, the flatness of the velocity increments $F(r)$ is computed from the velocity structure function of order $q: S_{q}(\boldsymbol{r})=\left\langle\left(\delta_{\boldsymbol{r}} \boldsymbol{u}\right)^{q}\right\rangle, F(\boldsymbol{r})=S_{4}(\boldsymbol{r}) / S_{2}(\boldsymbol{r})^{2}$ with $\delta_{\boldsymbol{r}} \boldsymbol{u}(\boldsymbol{x})=\boldsymbol{u}(\boldsymbol{x}+\boldsymbol{r}, t)-\boldsymbol{u}(\boldsymbol{x}, t)$. Figure 29(a) shows the comparison for the flatness computed from the longitudinal structure function of the normal velocity component for $R e_{*}=1000$ and $y^{+}=75$. One observes that for LES and LES-SSAM, the flatness remains close to the DNS. However, because the mesh used for LES and LES-SSAM is very coarse and is far larger than the Kolmogorov scale, the flatness stays of order of 3, corresponding to a Gaussian field. In contrast, to ensure numerical stability, the time step of the numerical simulation is lower than the Kolmogorov time scale 


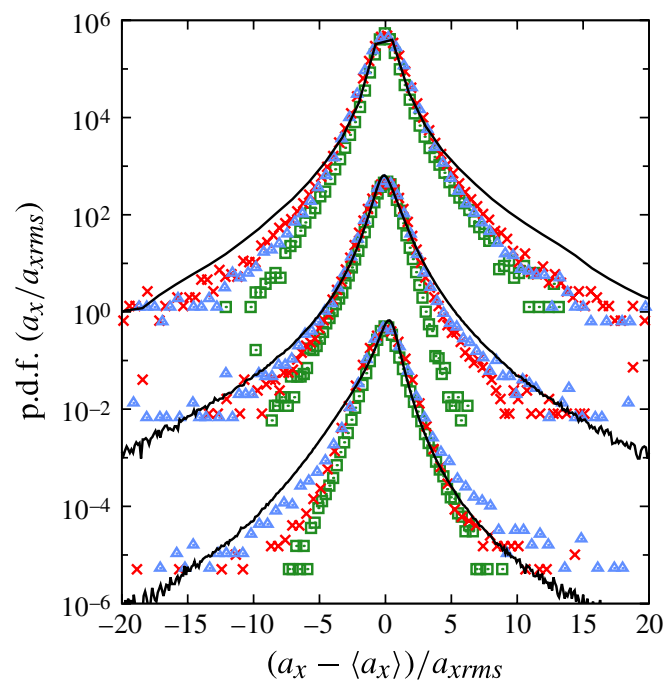

FIGURE 26. (Colour online) p.d.f. of longitudinal acceleration at $y^{+}=5,20$ and 950 respectively shifted upward by 3 decades, for $R e_{*}=1000$. Squares: LES; crosses: LES-SSAM-1; triangles: LES-SSAM-2; line: DNS.

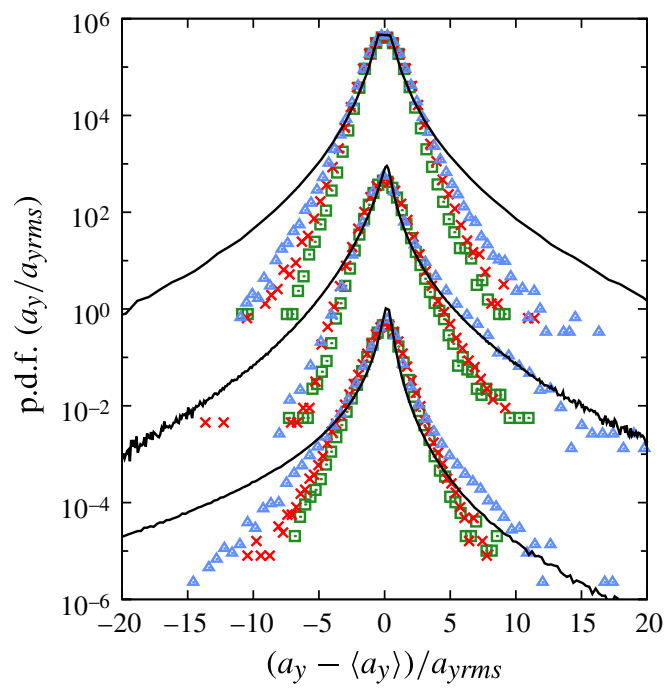

FIGURE 27. (Colour online) p.d.f. of wall-normal acceleration at $y^{+}=5,20$ and 950 respectively shifted upward by 3 decades, for $R e_{*}=1000$. Squares: LES; crosses: LES-SSAM-1; triangles: LES-SSAM-2; line: DNS.

(table 2). Therefore, we are interested in the temporal increments of the velocity at fixed positions. The flatness is computed from the Eulerian temporal structure function: $S_{q}(t)=\left\langle\left(\delta_{t} \boldsymbol{u}\right)^{q}\right\rangle, \quad F(t)=S_{4}(t) / S_{2}(t)^{2}$ with $\delta_{t} \boldsymbol{u}(\boldsymbol{x})=\boldsymbol{u}\left(\boldsymbol{x}, t^{\prime}+t\right)-\boldsymbol{u}\left(\boldsymbol{x}, t^{\prime}\right)$. In figure $29(b)$, the comparison for the flatness of the normal velocity temporal increments at $y^{+}=75$ and $R e_{*}=1000$ is given. In LES the velocity distribution 


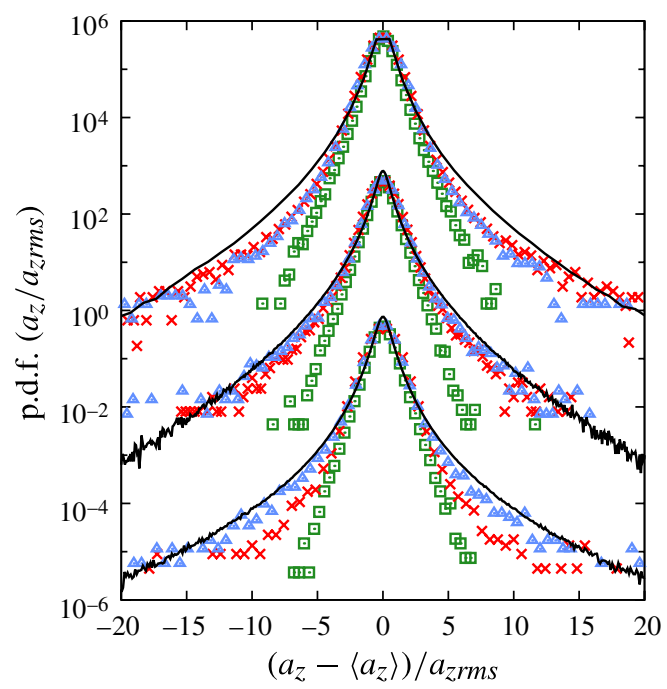

FIGURE 28. (Colour online) p.d.f. of transverse acceleration at $y^{+}=5,20$ and 950 respectively shifted upward by 3 decades, for $R e_{*}=1000$. Squares: LES; crosses: LES-SSAM-1; triangles: LES-SSAM-2; line: DNS.
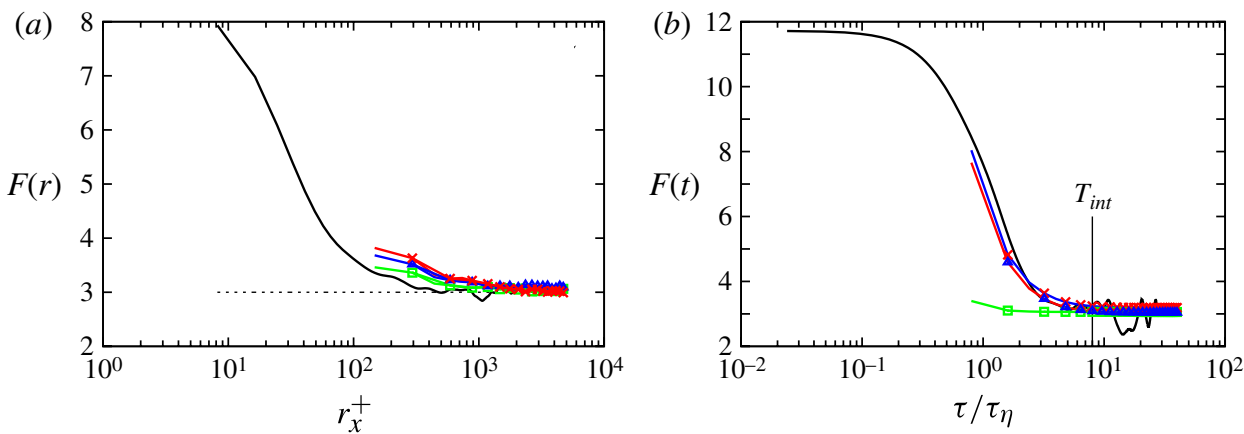

FIGURE 29. (Colour online) Evolution of the wall-normal velocity increment flatness at $y^{+}=75$ and $R e_{*}=1000$. (a) Evolution with spatial separation in the streamwise direction. The dashed line corresponds to a Gaussian field. $(b)$ Evolution with temporal separation $\left(T_{i n t}\right.$ is the integral time scale). Squares: LES; crosses: LES-SSAM-1; triangles: LES-SSAM-2; line: DNS.

remains close to Gaussian at all time lags, while in LES-SSAM, the flatness increases similarly to the DNS with decreasing time lag. Hence effects of intermittency associated with small scales are captured by LES-SSAM.

\subsection{Application of LES-SSAM to the transport of solid particles}

Because of the practical interest of particle-laden turbulent flows, a number of laboratory studies have been performed in order to assess the interaction between particles and the carrier turbulent flow. Among them, LES of inertial particle transport (Armenio, Piomelli \& Fiorotto 1999; Marchioli, Salvetti \& Soldati 2008; Wang \& Squires 1996) show that with a fine enough mesh, particle velocity statistics may reproduce acceptably the DNS data. However, in the case of a coarse grid, the 
gas velocity 'seen' by the particles is 'under-resolved'. As a result, the Lagrangian statistics of particle motion are simulated inaccurately, even for highly inertial particles. To obtain accurate statistics, usually LES is supplemented by a subgrid-scale modelling in the particle equation of motion (Kuerten 2006; Vinkovic et al. 2006; Marchioli et al. 2008; Pozorski \& Apte 2009). It has been shown in the previous section that LES-SSAM gives the unfiltered velocity and that the turbulent flow structure is significantly better predicted in comparison to standard LES. One may expect that prediction of particle dynamics could also be improved. The advantage here, is that additional modeling in the particle equation of motion (5.7) is unnecessary since LES-SSAM provides the instantaneous un-filtered velocity at the scale of the solid particle. An example of practical relevance is the 'turbophoresis' effect, where, due to the turbulent structures, particles move towards the walls. Kuerten \& Vreman (2005) and Kuerten (2006) reported that particle accumulation close to the wall may be significantly controlled by the small scales of the flow. The authors showed that standard LES fails to estimate correctly the particle concentration close to the wall. This motivated us to perform a comparison of particle statistics obtained by DNS, LES-SSAM and LES in the case of a channel flow with immersed spherical, non-rotating, heavy particles at low concentrations.

\subsubsection{Methodology}

It is assumed that the particle motion is governed by the Stokes law:

$$
\begin{aligned}
\frac{\mathrm{d} \boldsymbol{u}_{p}}{\mathrm{~d} t} & =-\frac{1}{\tau_{p}}\left(\boldsymbol{u}_{p}-\boldsymbol{u}\right) f\left(R e_{p}\right), \\
\frac{\mathrm{d} \boldsymbol{x}_{p}}{\mathrm{~d} t} & =\boldsymbol{u}_{p},
\end{aligned}
$$

where $\boldsymbol{u}_{p}$ and $\boldsymbol{x}_{p}$ are the velocity and position of a particle, and $\boldsymbol{u}$ is the carrier gas velocity at the particle position; $\tau_{p}=\rho_{p} d_{p}^{2} /(18 \rho v)$ is the response time of the particles, with $d_{p}$ the particle diameter. The Stokes number $S t=\tau_{p} / t_{*}$ is defined here by the ratio of the particle response time to a typical flow time scale $t_{*}=v / u_{*}^{2}$. The latter is analogous to the Kolmogorov time scale $t_{*}=\left(v / \varepsilon_{*}\right)^{1 / 2}$ in wall units. Action of the surrounding fluid, which is modelled by the drag force, takes into account the correction proposed by Clift, Grace \& Weber (1978): $f\left(R e_{p}\right)=1+0.15 R e_{p}^{0.687}$, where $\operatorname{Re}_{p}=d_{p}\left|\boldsymbol{u}_{p}-\boldsymbol{u}\right| / \nu$ is the local particle Reynolds number.

From (5.7)-(5.8), the fluid velocity is computed by DNS, LES and LES-SSAM. The fluid velocity at the particle position is then estimated by a third-order threedimensional Hermite interpolation scheme. Equations (5.7)-(5.8) are integrated by the Adams-Bashford method. The particle initial position is randomly chosen and its initial velocity is set to the fluid velocity. The wall is considered to be perfectly smooth. Each particle that strikes the wall rebounds elastically. Particle statistics are sampled starting from $t^{+}=t / t_{*} \approx 1000$, counted from particle release. For all simulations, these statistics correspond to the quasi-stationary state (Marchioli \& Soldati 2002). Five sets of 200000 particles are analysed, with different Stokes numbers, ranging from $S t=1$ to 125 , with a density ratio of $\rho_{p} / \rho=770$. The Reynolds number is $R e_{*}=590$ and the same mesh resolution as previously is used (table 2). The physical parameters of the particles are summarized in table 3.

We present the comparison of the particle statistics when the carrier phase is simulated by LES-SSAM, LES or DNS. The resolution of the velocity field by the LES-SSAM approach corresponds to the LES-SSAM-1 case. Although this model did 


$\begin{array}{cccc}R e_{*} & S t & d / l_{*} & \rho_{p} / \rho \\ 590 & 1 & 0.15 & 770 \\ 590 & 5 & 0.33 & 770 \\ 590 & 15 & 0.58 & 770 \\ 590 & 25 & 0.74 & 770 \\ 590 & 125 & 1.66 & 770\end{array}$

TABLE 3. Parameters used for the particle simulation $\left(l_{*}=v / u_{*}\right)$.

not match the DNS perfectly, it is simpler than LES-SSAM2 and thus we prefer to show the results from the model with fewer constants.

\subsubsection{Comparison with DNS and standard LES}

In figure 30, the particle velocity statistics are assessed. First, in figure $30(a)$, the mean particle velocity is compared for five Stokes numbers, $S t=1,5,15$, 25 and 125. In the logarithmic layer, for all five Stokes numbers considered, the particle velocity profile given by the LES-SSAM-1 is closer to the DNS than standard LES. For the standard deviations of the particle velocity components, the difference between LES and LES-SSAM is found essentially in the wall region. This is shown in figure $30(b-d)$. For the wall-normal component (figure $30 c$ ), which is mainly responsible for the cross-channel migration of particles, LES-SSAM is a fair improvement on the prediction of r.m.s. velocity. For the streamwise r.m.s. of particle velocity (figure $30 \mathrm{~b}$ ), the difference between LES and LES-SSAM is small, though with increasing Stokes number, the peak of r.m.s. values becomes broader for the LES, whereas in LES-SSAM this peak does not vary, as in DNS. For the spanwise component of particle velocity (figure 30d), LES-SSAM overestimates the r.m.s. values. This overestimation of the spanwise velocity r.m.s. has already been observed for the fluid phase (figure $21 b$ ). It is also seen that for $S t=125$, the difference in r.m.s. values obtained by DNS, LES and LES-SSAM is almost negligible. A particle, with a response time much larger than the Kolmogorov time is prone to 'filter' the flow structure on subgrid scales. At the same time, the mean flow velocity is better predicted by LES-SSAM. Consequently for $S t=125$ (figure 30a), LES-SSAM gives a better estimation of the mean longitudinal velocity of particles.

In figure 31, the profiles of particle mean concentration from the DNS, LES and LES-SSAM are shown for the five Stokes numbers. It is seen that modelling the flow acceleration at the discarded scales of the simulation can indeed contribute significantly to particle accumulation close to the wall. In contrast to LES, particle mean concentration provided by LES-SSAM matches quite well the DNS. Low-inertia particles do not sample intermittency of turbulent structures in the wall region. They tend to travel along with the surrounding fluid. Accordingly, it is seen in figure 31, that for $S t=1$, the effect of particle accumulation close to the wall is very small. Neither do highly inertial particles respond to this intermittency, due to the filtering effect. This is illustrated in figure 31 for $S t=125$. The profile of particle mean concentration appears to be almost uniform. Only particles with intermediate inertia are subjected to turbophoresis. In the considered range of Stokes numbers, the strongest accumulation of particles on the walls is obtained for $S t=15$. This is correctly reproduced by LES-SSAM, whereas, as reported by Kuerten \& Vreman (2005), standard LES fails in the prediction of particle mean concentration. 

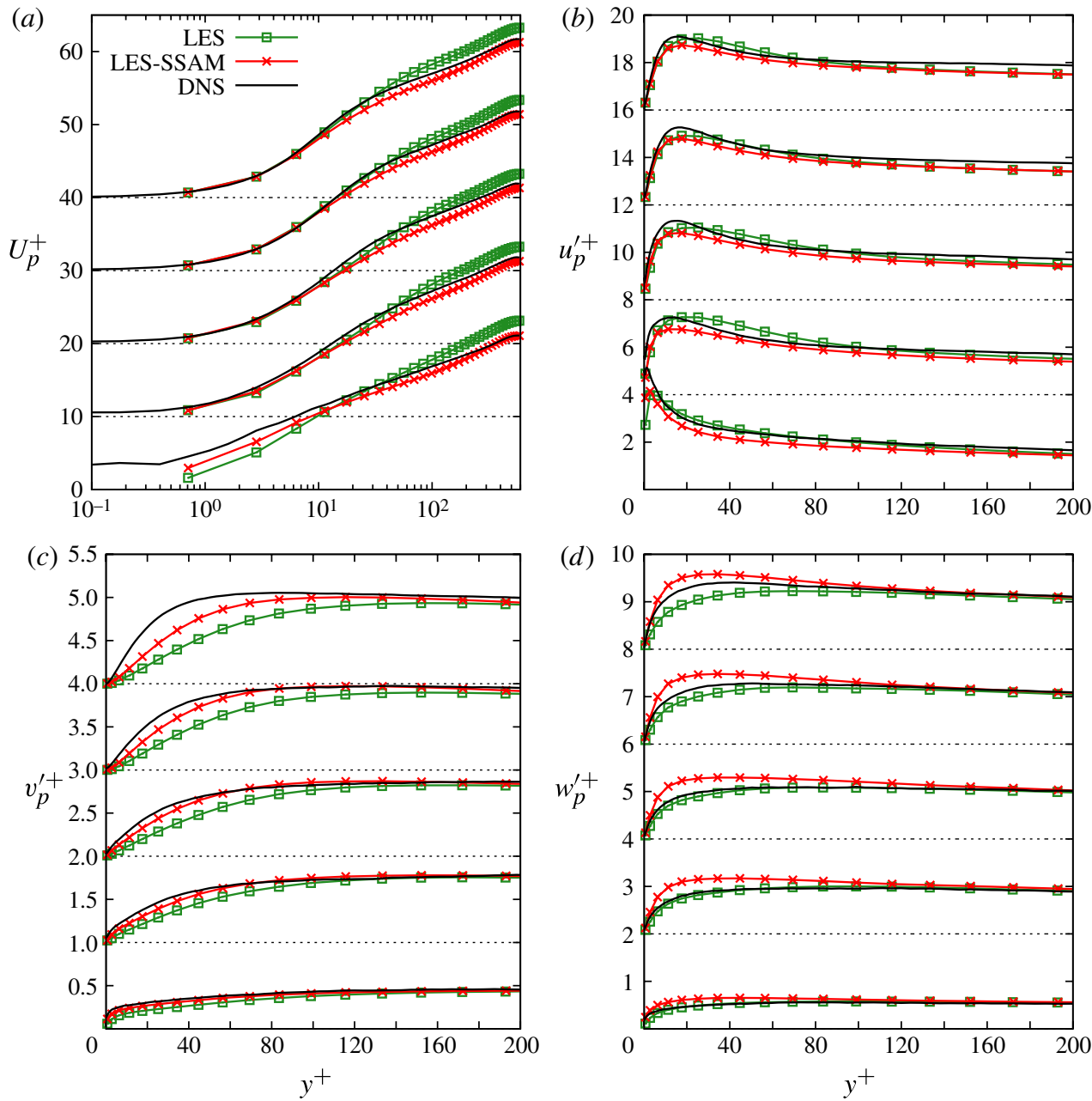

FIGURE 30. (Colour online) Profiles for particle velocity statistics at $R e_{*}=590$, for $S t=1$, 5, 15, 25 and 125 from top to bottom, respectively. Squares: LES; crosses: LES-SSAM; line: DNS. (a) Mean velocity (log-linear scales). r.m.s. acceleration profiles (zoom in $y^{+}<200$ region) for the streamwise $(b)$, the wall-normal $(c)$ and the spanwise $(d)$ component.

Acceleration of a solid particle is linked to its inertia and to the lifetime of turbulent structures interacting with the particle; thus one would expect an important influence of subgrid flow structure on the acceleration of particles with response times of the order of the Kolmogorov time. It is therefore interesting to complete our assessment of LES-SSAM by its prediction for the statistics of solid particle acceleration. This acceleration is computed from the derivative of the particle velocity. As proposed by Mordant et al. (2004b), the derivative is provided by the convolution with the derivative of a Gaussian kernel. This kernel ensures the reduction of errors due to numerical interpolation of the fluid velocity at the position of particles. As shown by Choi et al. (2004) these errors may lead to inaccurate estimation of particle acceleration. The filter cut-off is chosen to be of the order of the Kolmogorov time scale at the wall. The consistency of this method has been carefully checked. Namely, the acceleration of fluid particles computed in this way was compared 


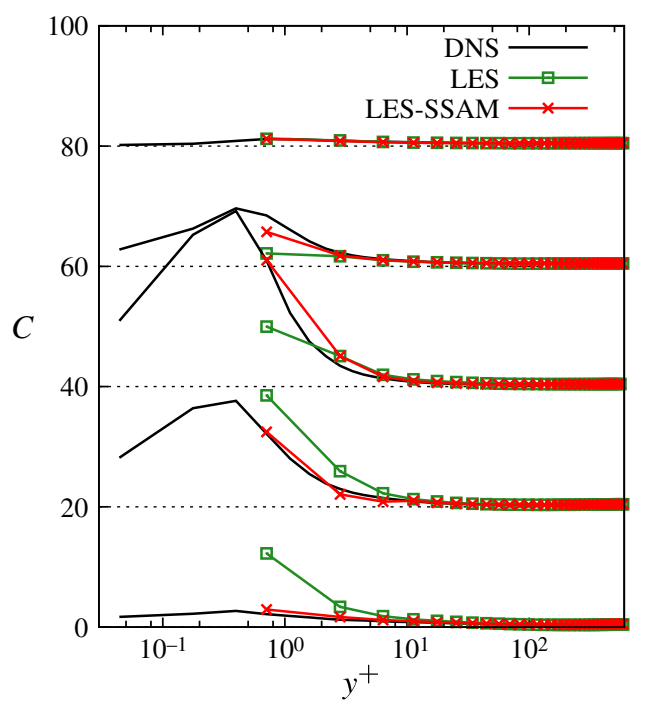

FIgURE 31. (Colour online) Particle mean concentration profile at $R e_{*}=590$, for $S t=1,5$, 15, 25 and 125, from top to bottom, respectively. Each profile is shifted by 20 units upward. Profiles are shown at $t^{+}=1200$ from the time when particles were released into the flow. Squares: LES; crosses: LES-SSAM; line: DNS.

with the Eulerian calculation of the material derivative. It was obtained that when a particle trajectory, with the chosen filter cut-off, is sampled every $0.06 t_{*}$, the mean, variance, skewness and flatness obtained from both approaches collapse. The r.m.s. values of three components of the particle acceleration are given in figure 32 for $S t=1,5,15,25$ and 125 . In the region up to $y^{+} \approx 100$, this figure shows clearly the contribution of subgrid scales with decreasing Stokes number, i.e. with decreasing particle response time. In the case of LES-SSAM, for all three components, the prediction of particle acceleration r.m.s. is getting closer to DNS, compared to standard LES. In contrast, particles with high Stokes number filter the subgrid flow structures. As result, the difference between LES and LES-SSAM becomes negligible. Very near the wall $\left(y^{+}<10\right)$ for $S t=15,25$ and 125 , one can observe a small peak in vertical acceleration r.m.s., growing slightly with increasing Stokes number. This is due to the condition on the wall for vertical particle velocity: a particle that strikes the wall rebounds elastically.

\section{Concluding remarks}

In the first part of this paper, the statistics of the total acceleration in turbulent channel flow are studied by DNS for three Reynolds numbers $\left(R e_{*}=180,590\right.$ and 1000). It has been observed that for each wall distance, the distribution of the acceleration norm follows a log-normal law with Reynolds-number-independent parameters: $\mu \approx(1 / 2) \sigma^{2}$ and $\sigma^{2} \approx \ln 2$. It is shown that the ratio of the mean norm of acceleration and the Kolmogorov acceleration $\left(\langle|a|\rangle / a_{\eta}\right)$ presents an evolution proportional to the mean velocity gradient, which may be related to interactions with the mean shear. Using the von Kármán hypothesis, the scaling law for $\langle|a|\rangle / a_{\eta}$ is found to be a logarithmic profile: $\langle|a|\rangle / a_{\eta} \approx \kappa \ln y^{+}$. While in line with the Barenblatt incomplete similarity, the profile $\langle|a|\rangle / a_{\eta}$ follows a power 

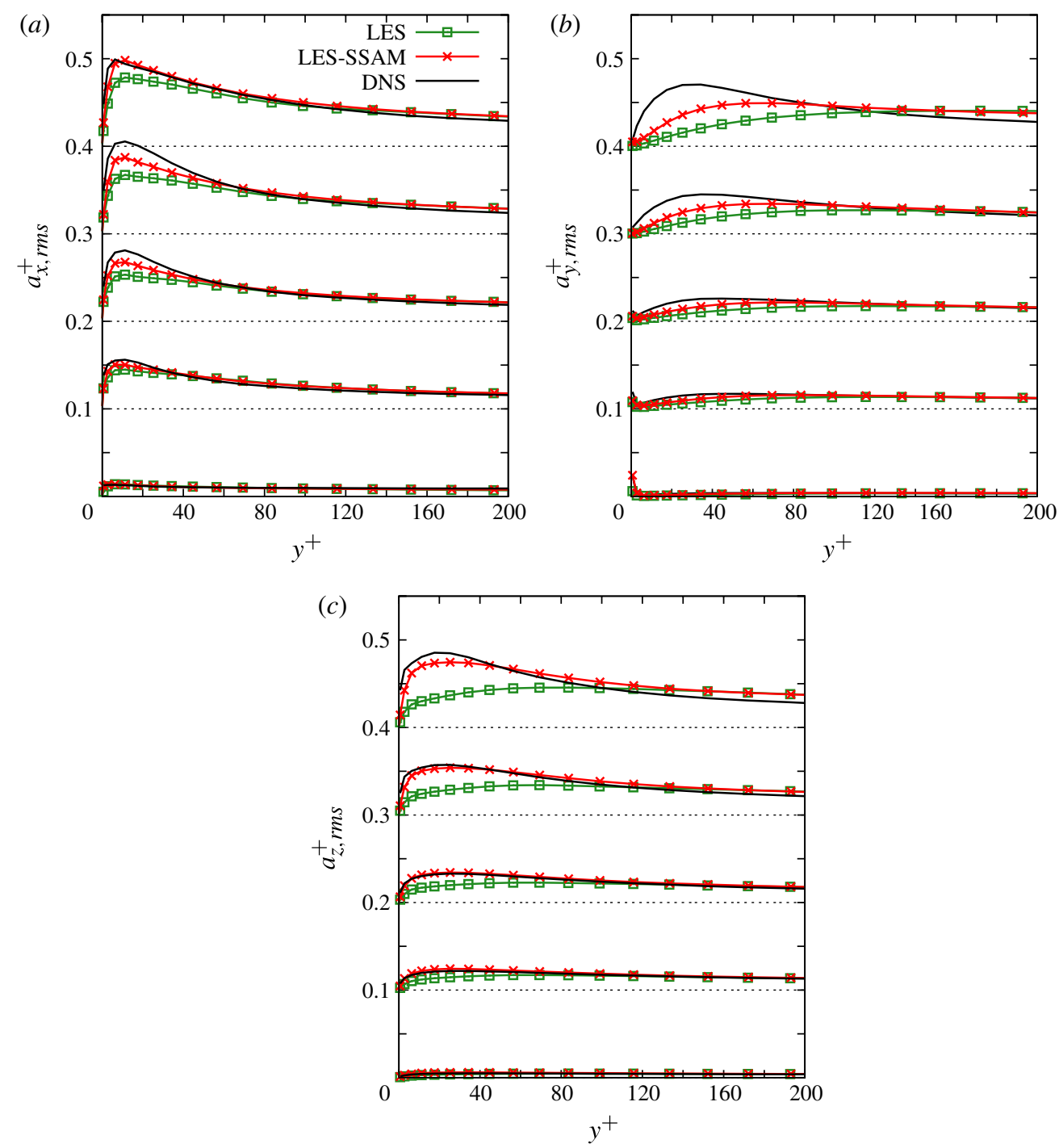

FIgURE 32. (Colour online) Particle r.m.s. acceleration profiles (zoom in $y^{+}<200$ region) for the streamwise $(a)$, the wall-normal $(b)$ and the spanwise $(c)$ component at $R e_{*}=590$, for $S t=1,5,15,25$ and 125, from top to bottom, respectively. Each profile has been shifted by 0.1 units upwards. Squares: LES; crosses: LES-SSAM; line: DNS.

law, with a logarithmic dependence on the Reynolds number of the parameters: $\langle|a|\rangle / a_{\eta}=\left(D_{0} \ln R e+D_{1}\right)\left(y^{+}\right)^{\beta_{1} / \ln (R e)}$. Although both laws are based on equally valid assumptions, the power law provides a better fit of the DNS profiles and its Reynoldsnumber dependence suggests that viscosity cannot be completely neglected, at least for Reynolds numbers lower than $R e_{*}=1000$. To emphasize the effect of the longrange interaction across the channel on the scaling, a logarithmic distance to the wall $\tau=\ln (h /(h-y))$ is introduced. With this evolution parameter it is observed that the mean acceleration norm can be described by a universal relation of only one parameter $\langle|a|\rangle \sim \ln \operatorname{Re}_{*} \Pi\left(\tau R e_{*}\right)$. Concerning its orientation, the acceleration relaxes towards 
isotropy with increasing wall distance. This relaxation appears to be independent of the Reynolds number. The quasi-isotropy is confirmed by the fact that far from the wall $\left(y^{+}>50\right)$, the statistics of the acceleration norm, conditioned on the orientation of acceleration, become almost independent of its orientation. Universality of these relations could be further investigated by studying other canonical wall flows (such as turbulent boundary layer or pipe flow) and by extending the Reynolds number range.

In the second part, stochastic models for the acceleration are introduced in the form of processes evolving across the channel with assumed forcing at the wall. The basic assumption is to consider the acceleration as being governed by two independent stochastic processes: one for the acceleration norm and the other for its orientation.

Two models are proposed for the norm, each based on statistical universalities in fragmentation under scaling symmetry, evolving across the channel with $\tau=$ $\ln (h /(h-y))$. The first model represents a 'non-self-averaging' stochastic process. The two parameters of the process are introduced as a function of the Reynolds number. This process has the log-normal distribution as intermediate asymptotic and further evolves towards a power-law distribution with increasingly stretched tails, which may represent ejections of small structures far from the wall. The second model represents a 'self-averaging' process. Consistently with our DNS observation, the distribution is relaxed to the log-normal law, with parameters estimated from homogeneous isotropic turbulence of the mean centreline acceleration norm. The second model is shown to represent very well the statistics of the acceleration norm. Comparing the two models, the relaxation towards the log-normal distribution may reflect the interactions of the inner region and the the 'external' turbulent stream.

In order to represent the relaxation towards isotropy, the model for the orientation is based on a random walk on a unit sphere evolving with distance to the wall. The DNS data show that the diffusion coefficient of this random walk is independent of the Reynolds number. Comparisons with DNS show that the model correctly reproduces the rate of return towards isotropy, although close to the wall, the Brownian motion fails to reproduce the large fluctuations of the orientation vector. It is worthwhile to note that a multifractal walk may help to recover the large fluctuations of $\theta$, but requires additional parameters to be fitted by DNS.

In the third part of this paper, the proposed stochastic models are coupled with largeeddy simulation in the LES-SSAM framework (Sabel'nikov et al. 2011), where the approximation of the 'unfiltered' instantaneous velocity field is given by a stochastic model equation. Comparisons of velocity, acceleration, and energy spectra between LES-SSAM, standard LES and DNS for $R e_{*}=590,1000$ and 2000 show that such a coupling can significantly improve the under-resolved simulations. Notably, LES-SSAM can enhance the vertical momentum flux, along with giving access to the small-scale intermittency of the flow. Furthermore, using either the first or the second acceleration norm models, LES-SSAM shows very similar results. This comparison implies that the region $10<y^{+}<100$, almost as well-predicted by the first model as by the second one, is a zone of significant interactions to be modelled. Further development of this approach is associated with numerous questions concerning more complex geometries, coupling between the resolved and subgrid fields, the structure of the acceleration field in the near-wall region. For example, spatio-temporal correlations between the large-scale field and the subgrid model can be introduced using parameters for the stochastic equation defined by the local properties of the resolved flow. The study by DNS of acceleration statistics conditioned on the velocity would provide useful information in that direction. Such correlations have been shown to exist in HIT (Hill 2002; Sawford et al. 2003; Crawford, Mordant \& Bodenschatz 
2005). In addition to that, the introduction of correlation between the norm and orientation of the acceleration near the wall may lead to a more accurate representation of wall structures.

The LES-SSAM approach, in addition to improving the statistics of the resolved field, has a practical advantage when physical phenomena are related to subgrid scales. To illustrate the importance of taking into account the unresolved acceleration, in the last part of the paper we focus on the transport of inertial particles in turbulent channel flow. The results show that, without modification of the particle equation of motion, LES-SSAM leads to significant improvement of the particle statistics in regard to LES and DNS. This is due to a more accurate resolution of the momentum redistribution in the flow. It has been shown that once the turbulence, 'seen' by particles, is better simulated, we achieve a better prediction of the particle migration towards the wall. This finding may open the possibility of applying the LES-SSAM successfully to other multiphase flows.

\section{Acknowledgements}

The authors would also like to express their gratitude to P. Moin, P. Spalart and V. Sabel'nikov for their useful comments on this work. M. Buffat is acknowledged for the development of the computational code. The authors acknowledge support from 'MAGIE' (Modélisation et approche générique de l'injection essence, with Continental Automotive France, and PSA). This work was granted access to the HPC resources of CINES under the allocation c201002560 made by GENCI (Grand Equipement National de Calcul Intensif). Numerical simulations were also performed on the P2CHPD cluster.

\section{REFERENCES}

Afonso, M. M. \& Meneveau, C. 2010 Recent fluid deformation closure for velocity gradient tensor dynamics in turbulence: timescale effects and expansions. Physica D 239, 1241-1250.

Antonia, R. A., Kim, J. \& Browne, L. W. B. 1991 Some characteristics of small-scale turbulence in a turbulent duct flow. J. Fluid Mech. 233, 369-388.

Armenio, V., Piomelli, U. \& Fiorotto, V. 1999 Effect of the subgrid scales on particle motion. Phys. Fluids 11 (10), 3030-3042.

Barenblatt, G. I. 1993 Scaling laws for fully developed turbulent shear flows. Part 1. Basic hypotheses and analysis. J. Fluid Mech. 248, 513-520.

Barenblatt, G. I. 1996 Scaling, Self-similarity, and Intermediate Asymptotics. Cambridge University Press.

Barenblatt, G. I. \& Prostokishin, V. M. 1993 Scaling laws for fully developed turbulent shear flows. Part 2. Processing of experimental data. J. Fluid Mech. 248, 521-529.

Brasseur, J. G. \& WeI, T. 2010 Designing large-eddy simulation of the turbulent boundary layer to capture law-of-the-wall scaling. Phys. Fluids 22 (2), 021303.

Buffat, M., Le Penven, L. \& Cadiou, A. 2011 An efficient spectral method based on an orthogonal decomposition of the velocity for transition analysis in wall bounded flow. Comput. Fluids 42, 62-72.

Burton, G. C. \& DAHM, W. J. A. $2005 a$ Multifractal subgrid-scale modeling for large-eddy simulation. I. Model development and a priori testing. Phys. Fluids 17, 075111.

Burton, G. C. \& DAHM, W. J. A. $2005 b$ Multifractal subgrid-scale modeling for large-eddy simulation. II. Backscatter limiting and a posteriori evaluation. Phys. Fluids 17, 075112.

Champagne, F. H., Harris, V. G. \& Corsin, S. 1970 Experiments on nearly homogeneous turbulent shear flow. J. Fluid Mech. 41, 81-139.

Chaouat, B. \& Schiestel, R. 2009 Further insight into sub-grid scale transport for continuous hybrid non-zonal RANS/LES simulations. In Proceeding of the Sixth Internal Symposium on 
Turbulence and Shear Flow Phenomena (TSFP 6) (ed. N. Kasagi, J. K. Eaton, J. A. C. Humphrey, A. V. Johansson \& H. J. Sung), pp. 1063-1068.

Chen, L., Coleman, S. W., Vassilicos, J. C. \& HU, Z. 2010 Acceleration in turbulent channel flow. J. Turbul. 11 (N41).

Chevillard, L. \& Meneveau, C. 2006 Recent fluid deformation closure for velocity gradient tensor dynamics in turbulence: timescale effects and expansions. Phys. Rev. Lett. 97, 174501.

Chol, J.-I., YeO, K. \& LEE, C. 2004 Lagrangian statistics in turbulent channel flow. Phys. Fluids 16 (3), 779-793.

Christensen, K. \& AdriAn, R. 2002 The velocity and acceleration signatures of small-scale vortices in turbulent channel flow. J. Turbul. 3, 27-29.

Clift, R., Grace, J. \& Weber, M. 1978 Bubble, Drops and Particles. Academic.

Crawford, A. M., Mordant, N. \& Bodenschatz, E. 2005 Joint statistics of the Lagrangian acceleration and velocity in fully developed turbulence. Phys. Rev. Lett. 94 (2), 024501.

Frisch, U. 1995 Turbulence: The Legacy of A. N. Kolmogorov. Cambridge University Press.

Germano, M., Piomelli, U., Moin, P. \& Cавот, W. H. 1991 A dynamic subgrid-scale eddy viscosity model. Phys. Fluids A 3 (7), 1760-1765.

GoROKHOVSKI, M. 2003 Fragmentation under the scaling symmetry and turbulent cascade with intermittency. In Annual Research Briefs 2003, pp. 197-203. Stanford University: Center for Turbulence Research.

Gorokhovski, M. A. \& Saveliev, V. L. 2008 Statistical universalities in fragmentation under scaling symmetry with a constant frequency of fragmentation. J. Phys. D: Appl. Phys. 41, 085405 .

HILl, R. J. 2002 Scaling of acceleration in locally isotropic turbulence. J. Fluid Mech. 452, 361-370.

HoYAs, S. \& JimÉNEZ, J. 2008 Reynolds number effects on the Reynolds-stress budgets in turbulent channels. Phys. Fluids 20 (10), 101511.

Hughes, T. J. R., Oberai, A. A. \& MAzzeI, L. 2001 Large eddy simulation of turbulent channel flows by the variational multiscale method. Phys. Fluids 13 (6), 1784-1799.

JimÉnez, J. 2012 Cascades in wall-bounded turbulence. Annu. Rev. Fluid Mech. 44, 27-45.

Kadanoff, L. P. 2000 Statistical Physics, Statics, Dynamics and Renormalization. World Scientific.

Kemenov, K. A. \& Menon, S. 2006 Explicit small-scale velocity simulation for high-Re turbulent flows. J. Comput. Phys. 220, 290-311.

Kemenov, K. A. \& Menon, S. 2007 Explicit small-scale velocity simulation for high-Re turbulent flows. Part II: Non-homogeneous flows. J. Comput. Phys. 222, 673-701.

Kerstein, A. R. 1999 One-dimensional turbulence: model formulation and application to homogeneous turbulence, shear flows, and buoyant stratified flows. J. Fluid Mech. 392, 277-334.

Kerstein, A. R. 2002 One-dimensional turbulence: a new approach to high-fidelity subgrid closure of turbulent flow simulations. Comput. Phys. Commun. 148, 1-16.

Kuerten, J. G. M. 2006 Subgrid modelling in particle-laden channel flow. Phys. Fluids 18, 025108 .

Kuerten, J. G. M. \& Vreman, A. W. 2005 Can turbophoresis be predicted by large-eddy simulation? Phys. Fluids 17, 011701.

Lamorgese, A. G., Pope, S. B., Yeung, P. K. \& Sawford, B. L. 2007 A conditionally cubic-Gaussian stochastic Lagrangian model for acceleration in isotropic turbulence. J. Fluid Mech. 582, 243-448.

Landau, L. D. \& Lifshitz, E. M. 1987 Fluid Mechanics, 2nd edn. Course of Theoretical Physics, Volume 6, Pergamon.

LARsson, J., Lien, F. S. \& YeE, E. 2007 Feedback-controlled forcing in hybrid LES/RANS. Intl J. Comput. Fluid Dyn. 20, 687-699.

LEe, C., YeO, K. \& CHOI, J.-I. 2004 Intermittent nature of acceleration in near-wall turbulence. Phys. Rev. Lett. 92 (14), 144502.

LÉvêque, E., Toschi, F., Shao, L. \& Bertoglio, J.-P. 2007 Shear-improved Smagorinsky model for large-eddy simulation of wall-bounded turbulent flows. J. Fluid Mech. 570, 491-502. 
LiLly, D. K. 1992 A proposed modification of the Germano subgrid-scale closure method. Phys. Fluids A 4 (3), 633-635.

Marchioli, C., Salvetti, M. V. \& Soldati, A. 2008 Some issues concerning large-eddy simulation of inertial particle dispersion in turbulent bounded flows. Phys. Fluids 20 (4), 040603.

Marchioli, C. \& Soldati, A. 2002 Mechanisms for particle transfer and segregation in a turbulent boundary layer. J. Fluid Mech. 468, 283-315.

Marusic, I., McKeon, B. J., Monkewitz, P. A., Nagib, H. M., Smits, A. J. \& SREENIVASAN, K. R. 2010 Wall-bounded turbulent flows at high Reynolds numbers: recent advances and key issues. Phys. Fluids 22 (6), 065103.

Meneveau, C. \& Katz, J. 2000 Scale-invariance and turbulence models for large-eddy simulation. Annu. Rev. Fluid Mech. 32, 1-32.

Monin, A. S. \& Yaglom, A. M. 1981 Statistical Fluid Mechanics: Mechanics of Turbulence, vol. 2. MIT.

Mordant, N., Crawford, A. M. \& Bodenschatz, E. 2004a Experimental Lagrangian acceleration probability density function measurement. Physica D 193 (1-4), 245-251.

Mordant, N., Crawford, A. M. \& Bodenschatz, E. $2004 b$ Three-dimensional structure of the Lagrangian acceleration in turbulent flows. Phys. Rev. Lett. 93 (21), 214501.

Mordant, N., Delour, J., Lévêque, E., Arnéodo, A. \& Pinton, J.-F. 2002 Long time correlations in Lagrangian dynamics: a key to intermittency in turbulence. Phys. Rev. Lett. 89 (25), 254502.

Moser, R. D., Kim, J. \& Mansour, N. N. 1999 Direct numerical simulation of turbulent channel flow up to $R e_{\tau}=590$. Phys. Fluids 11 (4), 943-945.

Moser, R. D., Moin, P. \& LeOnARD, A. 1983 A spectral numerical method for the Navier-Stokes equations with applications to Taylor-Couette flow. J. Comput. Phys. 52, 524-544.

PARK, N. \& MAHESH, K. 2008 A velocity-estimation subgrid model constrained by subgrid scale dissipation. J. Comput. Phys. 227, 4190-4206.

Pinsky, M., Khain, A. \& Tsinober, A. 2000 Accelerations in isotropic and homogeneous turbulence and Taylor's hypothesis. Phys. Fluids 12 (12), 3195-3204.

Piomelli, U. \& Balaras, E. 2002 Wall-layer models for large-eddy simulations. Annu. Rev. Fluid Mech. 34, 349-374.

Piomelli, U., Balaras, E., Pasinato, H., Squires, K. D. \& Spalart, P. R. 2003 The inner-outer layer interface in large-eddy simulations with wall-layer models. Intl J. Heat Fluid Flow 24 (4), 538-550.

Pope, S. B. 1990 Lagrangian microscales in turbulence. Phil. Trans. R. Soc. Lond. 333 (1631), 309-319.

Pope, S. B. 1991 Application of the velocity-dissipation probability density function model to inhomogeneous turbulent flows. Phys. Fluids A 3 (8), 1947-1957.

Pope, S. B. 2000 Turbulent Flows. Cambridge University Press.

Pope, S. B. \& Chen, Y. L. 1990 The velocity-dissipation probability density function model for turbulent flows. Phys. Fluids 2 (8), 1437-1449.

Pozorski, J. \& APTE, S. V. 2009 Filtered particle tracking in isotropic turbulence and stochastic modelling of subgrid-scale dispersion. Intl J. Multiphase Flow 35 (2), 118-128.

Reynolds, A. M., Mordant, N., Crawford, A. M. \& Bodenschatz, E. 2005 On the distribution of Lagrangian accelerations in turbulent flows. New J. Phys. 7 (1), 58.

SABel'nikov, V., Chtab-Desportes, A. \& Gorokhovski, M. 2011 New sub-grid stochastic acceleration model in LES of high-Reynolds-number flows. Eur. Phys. J. B 80 (2), 177-187.

SAGAUT, P. 2002 Large Eddy Simulation for Incompressible Flows: An Introduction, 2nd edn. Springer.

Sagaut, P., Montreuil, E. \& Labbé, O. 1999 Assessment of some self-adaptive SGS models for wall bounded flows. Aerosp. Sci. Technol. 6, 335-344.

Sarghini, F., Piomelli, U. \& Balaras, E. 1999 Scale-similar models for large-eddy simulations. Phys. Fluids 11 (6), 1596-1607.

Saveliev, V. L. \& Gorokhovski, M. A. 2005 Group-theoretical model of developed turbulence and renormalization of the Navier-Stokes equation. Phys. Rev. E 72, 016302. 
SAVeliev, V. L. \& Gorokhovski, M. A. 2012 Renormalization of the fragmentation equation: exact self-similar solutions and turbulent cascades. Phys. Rev. E 86, 061112.

Sawford, B. L., Yeung, P. K., Borgas, M. S., Vedula, P., La Porta, A., Crawford, A. M. \& BodenschatZ, E. 2003 Conditional and unconditional acceleration statistics in turbulence. Phys. Fluids 15 (11), 3478-3489.

Schmidt, R. C., Kerstein, A. R. \& Wunsch, S. 2003 Near-wall LES closure based on one-dimensional turbulence modelling. J. Comput. Phys. 186, 317-355.

Shur, M. L., Spalart, P. R., Strelets, M. Kh. \& Travin, A. K. 2008 A hybrid RANS-LeS approach with delayed-DES and wall-modelled LES capabilities. Intl J. Heat Fluid Flow 29 (6), 1638-1649.

Smits, A, J., McKeon, B. J. \& Marusic, I. 2011 High-Reynolds Number Wall Turbulence. Annu. Rev. Fluid Mech. 43, 353-375.

Spalart, P. R., Deck, S., Shur, M. L., Squires, K. D., Strelets, M. Kh. \& Travin, A. 2006 A new version of detached-eddy simulation, resistant to ambiguous grid densities. Theor. Comput. Fluid Dyn. 20, 181-195.

Toschi, F. \& Bodenschatz, E. 2009 Lagrangian properties of particles in turbulence. Annu. Rev. Fluid Mech. 41 (1), 375-404.

Tsinober, A., Vedula, P. \& Yeung, P. K. 2001 Random taylor hypothesis and the behaviour of local and convective accelerations in isotropic turbulence. Phys. Fluids 13 (7), 1974-1984.

Vedula, P. \& Yeung, P. K. 1999 Similarity scaling of acceleration and pressure statistics in numerical simulations of isotropic turbulence. Phys. Fluids 11 (5), 1208-1220.

Vinkovic, I., Aguirre, C., Ayrault, M. \& Simoëns, S. 2006 Large-eddy simulation of the dispersion of solid particles in a turbulent boundary layer. Boundary-Layer Meteorol. 121, 283-311.

Volker, S., Moser, R. D. \& Venugopal, P. 2002 Optimal large eddy simulation of turbulent channel flow based on direct numerical simulation statistical data. Phys. Fluids 14 (10), 3675-3691.

Voth, G. A., La Porta, A., Grawford, A. M., Alexander, J. \& Bodenschatz, E. 2002 Measurements of particle accelerations in fully developed turbulence. J. Fluid Mech. 469, 121.

Voth, G. A., Satyanarayan, K. \& Bodenschatz, E. 1998 Lagrangian acceleration measurements at large Reynolds numbers. Phys. Fluids 10, 2268.

WANG, Q. \& SQUiRES, K. D. 1996 Large eddy simulation of particle-laden turbulent channel flow. Phys. Fluids 8 (5), 1207-1223.

Westbury, P. S., Dunn, D. C. \& Morrison, J. F. 2004 Analysis of a stochastic backscatter model for the large-eddy simulation of wall-bonded flow. Eur. J. Mech. B 23, 737-758.

Xu, H., Ouellette, N. T., Vincenzi, D. \& Bodenschatz, E. 2007 Acceleration correlations and pressure structure functions in high-Reynolds number turbulence. Phys. Rev. Lett. 99, 204501.

Yeo, K., Kim, B.-G. \& LEE, C. 2010 On the near-wall characteristics of acceleration in turbulence. J. Fluid Mech. 659, 405-419.

Yeung, P. K., Pope, S. B., LAmorgese, A. G. \& Donzis, D. A. 2006 Acceleration and dissipation statistics of numerically simulated isotropic turbulence. Phys. Fluids 18, 065103.

ZAMANSKY, R., VinKovic, I. \& GoROKHOVSKI, M. 2010 LES approach coupled with stochastic forcing of subgrid acceleration in a high Reynolds number channel flow. J. Turbul. 11 (30), $1-18$. 